DFPD-04/TH-01

SISSA $4 / 2004 / \mathrm{EP}$

\title{
Lepton Flavor Violation, Neutralino Dark Matter and the Reach of the LHC
}

\author{
A. Masiero ${ }^{1}$, S. Profumo ${ }^{2}$, S. K. Vempati ${ }^{1}$ and C. E. Yaguna ${ }^{2}$ \\ 1 Dipartimento di Fisica 'G. Galilei', Università di Padova, and Istituto Nazionale di \\ Fisica Nucleare, Sezione di Padova, Via Marzolo 8, I-35131, Padova, Italy \\ 2 Scuola Internazionale Superiore di Studi Avanzati, Via Beirut 2-4, I-34014 Trieste, \\ Italy and Istituto Nazionale di Fisica Nucleare, Sezione di Trieste, I-34014 Trieste, Italy \\ E-mail: antonio.masiero@pd.infn.it, profumo@sissa.it, \\ sudhir.vempati@pd.infn.it, yaguna@sissa.it
}

\begin{abstract}
We revisit the phenomenology of the Constrained MSSM with right-handed neutrinos (CMSSMRN). A supersymmetric seesaw mechanism, generating neutrino masses and sizable lepton flavour violating (LFV) entries is assumed to be operative. In this scheme, we study the complementarity between the 'observable ranges' of various paths leading to the possible discovery of low energy SUSY: the reach of the Cern Large Hadron Collider (LHC), the quest for neutralino dark matter signals and indirect searches through LFV processes. Within the regions of the CMSSMRN parameter space compatible with all cosmo-phenomenological requirements, those which are expected to be probed at the LHC will be typically also accessible to upcoming LFV experiments. Moreover, parameter space portions featuring a heavy SUSY particle spectrum could be well beyond LHC reach while leaving LFV searches as the only key to get a glimpse on SUSY.
\end{abstract}




\section{Introduction}

This is going to be the decade where we should be able to establish whether low energy supersymmetry (SUSY) exists or not. We have three main roads to gain access to SUSY: (a) Direct SUSY searches at hadron colliders (b) Indirect SUSY searches in rare FCNC (Flavour Changing Neutral Currents) and/or CP violating processes (c) Direct and indirect SUSY Dark Matter (DM) searches. Given that low energy supersymmetry is rather a framework than a predictive model, one has to assume an explicit realisation of SUSY breaking in order to make predictions concerning the above mentioned three roads to SUSY. The Constrained Minimal SUSY Standard Model (CMSSM) represents the 'prototype' of low energy supergravity having the minimal number of free parameters and being successful in not departing too violently from the Standard Model (SM) predictions. In view of the extraordinary agreement of the SM with all the experimental data on the above (a) and (b) points, it is of interest to consider models with features approaching those of the CMSSM.

The CMSSM, in its original formulation, is however not complete, and it has to be supplemented with some mechanism to generate non-zero neutrino masses and mixings. The supersymmetric seesaw mechanism represents a very attractive candidate to accomplish this task. Hence we are led to study a CMSSM with right handed neutrinos (CMSSMRN). We consider this as the 'minimal' low-energy supergravity-inspired setting with massive neutrinos. Compared to the CMSSM, the main novelty in the CMSSMRN is the presence of lepton flavour violation (LFV) [1]. Information from neutrino masses is nonetheless not sufficient to determine all the seesaw parameters 2], which are crucial to compute the relevant LFV rates. To remedy this, either a top-down approach with specific SUSYGUT models and/or flavour symmetries 3, 4, 5, 6, or a bottom-up approach with specific parameterisations of low energy unknowns have been adopted in the literature [7, 8, 9, In the present work, we follow a hybrid approach making the minimal assumptions required to successfully predict lepton flavour violation. Assuming one of the neutrino Yukawa couplings to be as large as the top Yukawa, and varying the other relevant seesaw parameters, we move from situations where LFV rates are significantly large, and already constrain the CMSSMRN parameter space (what we call the "best-case" scenario) to cases where a significant experimental improvement would be needed to detect flavour violation in the lepton sector ("worst-case" scenario).

In the light of this setting, we can now revisit the above three roads leading to SUSY discovery. Namely, we wish to conduct a quantitative analysis taking into consideration (a) direct search limits for SUSY at LEP and hadron colliders (b) indirect searches in rare FCNC processes, particularly concentrating on LFV, and finally (c) the neutralino relic density, which should respect the upper bound on the cold dark matter (CDM) content of the universe. We outline a strong complementarity between the three mentioned roads for SUSY discovery. We generically find, in the "best case" scenario, that the inclusion of the LFV constraints significantly restricts the already "Constrained" MSSM parameter space. In particular, in the most favorable cases, if stau coannihilations are responsible for the suppression of the neutralino relic density, SUSY might 'appear' in LFV experiments even before the advent of the LHC, which will thoroughly probe these regions. In the other two parameter space areas allowed by the stringent WMAP upper bound [10] on 
the CDM content, namely the A-pole funnel and focus point regions, LFV sensitivity is found to be usually larger than the LHC reach, provided the unknown leptonic mixing angle $U_{e 3}$ is not too small. Indeed, given that, particularly in the focus point region, SUSY masses are quite heavy, it might well be that LFV constitutes the only tool at our disposal to get SUSY signals. Therefore, in the "best-case" situation, the inclusion of massive neutrinos has critical implications on the discovery paths for SUSY. On the other hand, in the "worst-case" scenario, LFV experimental studies will at best only be complementary to LHC direct SUSY searches. In this case, non-zero neutrino masses do not have a significant impact on the CMSSMRN phenomenology, which would be almost indistinguishable from that of the CMSSM.

Some interesting analyses of the impact of LFV on the CMSSM parameter space already exist: for instance, in Ref. [1] the correlation between DM and LFV was considered in a bottom-up approach. Recently, in Ref. [12] the connections between rare processes and seesaw generated LFV in CMSSM have been studied. Our analysis simultaneously takes into account high energy collider physics and low energy flavour constraints as well as the latest information on the DM content of the universe. Moreover, the hybrid approach on the seesaw implementation that we adopt here differs from what was done in previous works. We detail our results in the following Sections.

In the next Section, we review the CMSSMRN model and present our assumptions. We also briefly review the present status of 'discovery limits' of SUSY within the three discovery methodologies. In Sections 3 and 4, we provide full details on our numerical results, mostly concentrating on the "best case" scenario. In Section 5, we stress the role of $U_{e 3}$ in connection with direct and indirect SUSY searches. After a brief summary of the results, we conclude with an outlook.

\section{The CMSSMRN and LFV}

The CMSSM is based on minimal supergravity with universal soft terms at the GUT scale, $M_{G U T} \approx 2 \cdot 10^{16} \mathrm{GeV}$. The SUSY particles spectrum at the weak scale is determined, through Renormalisation Group ( $\mathrm{RG}$ ) evolution, by specifying the following high scale parameters: $m_{0}$, the common scalar mass, $A_{0}$, the common trilinear coupling and $M_{1 / 2}$, the universal gaugino mass. The remaining low energy parameters of the model are $\tan \beta$, the ratio of the Higgs vacuum expectation values (vev) and the sign of $\mu$. The superpotential of the CMSSM incorporating the seesaw mechanism [13] can be written as

$$
W=W_{Y_{Q}}+h_{i j}^{e} L_{i} e_{j}^{c} H_{1}+h_{i j}^{\nu} L_{i} \nu_{j}^{c} H_{2}+M_{R_{i j}} \nu_{i}^{c} \nu_{j},
$$

where the leptonic part has been detailed, while the quark Yukawa couplings and the $\mu$ parameter are contained in $W_{Y_{Q}}$. $i, j$ are generation indices. $M_{R}$ represents the (heavy) Majorana mass matrix for the right-handed neutrinos. Eq.(11) leads to the standard seesaw formula for the (light) neutrino mass matrix

$$
\mathcal{M}_{\nu}=-h^{\nu} M_{R}^{-1} h^{\nu T} v_{2}^{2}
$$

where $v_{2}$ is the vacuum expectation value (VEV) of the up-type Higgs field, $H_{2}$. Under suitable conditions on $h^{\nu}$ and $M_{R}$ the correct mass splittings and mixing angles in $\mathcal{M}_{\nu}$ 
can be obtained. Detailed analyses deriving these conditions are already present in the literature [14. Additionally, the decoupled heavy right handed neutrinos also leave their imprints on the soft sector in terms of flavour violating entries [1] which can be probed in LFV experiments.

The amount of lepton flavour violation generated by the SUSY seesaw crucially depends on the flavour structure of $h^{\nu}$ and $M_{R}$, the new sources of flavour violation which do not appear in the CMSSM. This dependence can be clearly seen in the Renormalisation Group-induced entries in the slepton mass matrices at the weak scale:

$$
\left(m_{\tilde{L}}^{2}\right)_{i j} \approx-\frac{3 m_{0}^{2}+A_{0}^{2}}{8 \pi^{2}} \sum_{k}\left(h_{i k}^{\nu} h_{j k}^{\nu *}\right) \ln \frac{M_{G U T}}{M_{R_{k}}}, \quad i \neq j,
$$

where $M_{R}$ is the scale of the right handed neutrinos. From above it is obvious that if either the neutrino Yukawa couplings or the flavour mixings present in $h^{\nu}$ are very tiny, the strength of LFV will be significantly reduced. Given that there are several possible regions in the seesaw parameter space which may generate the observed mixing in the neutrino sector, one has to resort to some assumptions on $h^{\nu}$ and on $M_{R}$ to make a quantitative analysis of LFV within this model.

In the present work, we take the view that the presence of non-zero neutrino masses modifies the CMSSM in such a way that the operative seesaw mechanism significantly maximises LFV leading, in the near future, to another sound discovery road for SUSY. To this extent, let us make the following assumptions [15, 6, 16]:

(a) At least one of the neutrino Yukawa couplings is of $\mathcal{O}(1)$. For definiteness, we choose it to be the third eigenvalue, $h_{3}^{\nu}$, setting it equal to the top quark Yukawa, $h_{t}$. Assuming neutrino masses are hierarchical, this automatically sets the largest eigenvalue of $M_{R}$ to be rather close to $M_{G U T}$, around $10^{14} \div 10^{15} \mathrm{GeV}$. The second largest and the smallest eigenvalues $h_{2,1}^{\nu}$ can be left unspecified as far as the dominant contribution to lepton flavour violation is concerned. However, for a complete neutrino mass model, one needs to specify these eigenvalues too.

(b) The matrix which diagonalises the product $h^{\nu} h^{\nu \dagger}$ has either a CKM-like structure with small mixing or a MNS like structure with large mixing. Notice that the mixing present in $h^{\nu} h^{\nu} \dagger$ determines the amount of LFV, as given by Eq. (33). Together with assumption (a), the case with small CKM-like mixing gives a "worst case" scenario, whereas the case with large MNS-like mixing provides a "best case" for LFV.

As far as neutrino masses and mixings are concerned, assumptions (a) and (b) have been extensively studied in the literature, and it has been shown that they may lead to phenomenologically viable models for neutrino masses [6]. The relevant references can be found in the reviews listed in Ref. [14]. Given these assumptions, the LFV mass-insertions at the weak scale in the CMSSMRN are given, for the worst and best case respectively as: 
Worst case:

$$
\begin{aligned}
& \left(m_{\tilde{L}}^{2}\right)_{21} \approx-\frac{3 m_{0}^{2}+A_{0}^{2}}{8 \pi^{2}} h_{t}^{2} V_{t d} V_{t s} \ln \frac{M_{G U T}}{M_{R_{3}}}+\mathcal{O}\left(h_{2}^{\nu}\right)^{2}, \\
& \left(m_{\tilde{L}}^{2}\right)_{32} \approx-\frac{3 m_{0}^{2}+A_{0}^{2}}{8 \pi^{2}} h_{t}^{2} V_{t b} V_{t s} \ln \frac{M_{G U T}}{M_{R_{3}}}+\mathcal{O}\left(h_{2}^{\nu}\right)^{2}, \\
& \left(m_{\tilde{L}}^{2}\right)_{31} \approx-\frac{3 m_{0}^{2}+A_{0}^{2}}{8 \pi^{2}} h_{t}^{2} V_{t b} V_{t d} \ln \frac{M_{G U T}}{M_{R_{3}}}+\mathcal{O}\left(h_{2}^{\nu}\right)^{2} .
\end{aligned}
$$

Best case:

$$
\begin{aligned}
& \left(m_{\tilde{L}}^{2}\right)_{21} \approx-\frac{3 m_{0}^{2}+A_{0}^{2}}{8 \pi^{2}} h_{t}^{2} U_{e 3} U_{\mu 3} \ln \frac{M_{G U T}}{M_{R_{3}}}+\mathcal{O}\left(h_{2}^{\nu}\right)^{2}, \\
& \left(m_{\tilde{L}}^{2}\right)_{32} \approx-\frac{3 m_{0}^{2}+A_{0}^{2}}{8 \pi^{2}} h_{t}^{2} U_{\mu 3} U_{\tau 3} \ln \frac{M_{G U T}}{M_{R_{3}}}+\mathcal{O}\left(h_{2}^{\nu}\right)^{2}, \\
& \left(m_{\tilde{L}}^{2}\right)_{31} \approx-\frac{3 m_{0}^{2}+A_{0}^{2}}{8 \pi^{2}} h_{t}^{2} U_{e 3} U_{\tau 3} \ln \frac{M_{G U T}}{M_{R_{3}}}+\mathcal{O}\left(h_{2}^{\nu}\right)^{2},
\end{aligned}
$$

where $V$ is the CKM matrix and $U$ is the leptonic 'PMNS' mixing matrix. It is now obvious that the seesaw generated mass insertions above lead to various LFV processes, like rare leptonic radiative decays, $\mu \rightarrow e$ conversion in nuclei [17, $\tau \rightarrow 3 \mu$ [18. One of the features which stands out is that in the best case scenario, the amplitude for any LFV process involving $\mu \rightarrow e$ transitions depends on the neutrino mixing angle, $U_{e 3}$, of which very little experimental information is known, except for an upper bound (See for ex: Ref. [4). The same statement is also true for $\tau \rightarrow e$ transitions. The $\tau \rightarrow \mu$ transitions are instead $U_{e 3}$-independent probes of SUSY, whose importance was first pointed out in Ref. [19]. Otherwise, the leptonic flavour violating transitions are now completely determined only in terms of the CMSSMRN parameters.

To appreciate the phenomenological impact of these rare transitions, we list below the present and upcoming experimental limits on the $l_{j} \rightarrow l_{i}, \gamma$ decays.

Present limits:

$$
\begin{array}{lll}
B R(\mu \rightarrow e \gamma) & \leq 1.2 \times 10^{-11} \\
B R(\tau \rightarrow \mu \gamma) & \leq 3.1 \times 10^{-7} \\
B R(\tau \rightarrow e \gamma) & \leq 3.7 \times 10^{-7}
\end{array}
$$

Upcoming limits:

$$
\begin{array}{lll}
B R(\mu \rightarrow e \gamma) & \leq 10^{-13} \div 10^{-14} & {[23]} \\
B R(\tau \rightarrow \mu \gamma) & \leq 10^{-8} & {[22]} \\
B R(\tau \rightarrow e \gamma) & \leq 10^{-8} & {[22]}
\end{array}
$$

In the next Sections, we will apply these bounds to constrain the CMSSMRN parameter space. 


\subsection{The Three Roads}

The seesaw mechanism operative within our model does not modify the SUSY mass spectrum of the CMSSM significantly. Thus except for LFV and other rare processes, the phenomenology of the CMSSM is very similar to that of the CMSSMRN. Let us briefly summarise the status of the mentioned three roads and spell out the various constraints we consider in the present work.

(i) Collider Searches: Direct collider searches will give conclusive evidences for the existence of low energy supersymmetry. The searches conducted at LEP already provide strong bounds on the CMSSM parameter space. The main constraints are [24]:

(a) The SM-like light Higgs boson mass:

$$
m_{h} \geq 114.1 \mathrm{GeV}, \text { and }
$$

(b) The lightest chargino mass:

$$
m_{\chi_{1}^{ \pm}} \geq 103 \mathrm{GeV}
$$

These bounds significantly constrain the CMSSM parameter space, especially in the low $\tan \beta$ and low mass spectrum ("bulk") region. Soon, more extended CMSSM regions will also be probed by the Fermilab Tevatron ${ }^{1}$. A by far larger portion of the CMSSM parameter space will be probed at the CERN Large Hadron Collider (LHC) ${ }^{2}$.

(ii) Rare processes: Low-energy supersymmetry can also manifest itself in various flavour and CP violating processes (see [27] for a review). In the CMSSM, where for most of the processes there is no significant deviation from the SM predictions, the main constraint on the parameter space stems from $B R(b \rightarrow s, \gamma)$ [28] (see also [29] for recent progresses). At present, combining the theoretical and experimental uncertainties, this constraint is given by 30]:

$$
2.16<B R(b \rightarrow s \gamma) \times 10^{4}<4.34
$$

The other main constraints come from the SUSY contributions to the anomalous magnetic moment of the muon, $\delta a_{\mu}$, and from the Higgs mediated $B_{s} \rightarrow \mu^{+} \mu^{-}$. It is known that the anomalous magnetic moment of the muon is capable of putting severe constraints on the supersymmetric parameter space. However, at present there are large theoretical and experimental uncertainties involved in evaluating the SM contribution to $\delta a_{\mu}$. The other significant constraint might come from the upper limit on the $B R\left(B_{s} \rightarrow \mu^{+} \mu^{-}\right)$from Tevatron [31, 32], but again as far as the CMSSM is concerned, $B R(b \rightarrow s, \gamma)$ is mostly

\footnotetext{
${ }^{1}$ It has been recently shown that parameter space up to $m_{0} \leq 200 \mathrm{GeV}$ and $M_{1 / 2} \leq 250 \mathrm{GeV}$ could be probed for a low $\tan \beta$ value assuming that the integrated luminosity of the Tevatron reaches $25 \mathrm{fb}^{-1}$ [25.

${ }^{2}$ Here, in terms of $m_{0}$ and $M_{1 / 2}$, the maximal region covered by LHC, at an integrated luminosity of $\sim 100 \mathrm{fb}^{-1}$, will be up to $1400 \mathrm{GeV}$ in $M_{1 / 2}$ for $m_{0} \leq 1 \mathrm{TeV}$. At larger $m_{0} \gtrsim 5 \mathrm{TeV}$, the maximal reach in $M_{1 / 2}$ will instead be around $700 \mathrm{GeV}$ [26].
} 
a much stronger bound. We postpone the consideration of these effects to a future work.

(iii) Neutralino Relic Density and Dark Matter Searches: In the CMSSM the lightest neutralino $\tilde{\chi}_{1}$ is the lightest supersymmetric particle (LSP) over a very wide range of parameters, providing an ideal particle candidate for cold dark matter. $\tilde{\chi}_{1}$ is practically always, to a high degree of purity, a bino, except for a thin strip close to the parameter space area where radiative electroweak supersymmetry breaking conditions are not fulfilled, named focus point region 33 .

The current "state-of-the-art" of supersymmetric dark matter studies faces on the one side an impressive progress in observational cosmology, which recently led to an unprecedented accurate determination of the amount of cold dark matter in the universe 10]:

$$
\Omega_{\mathrm{CDM}} h^{2}=0.1126_{-0.009}^{+0.008},
$$

where $\Omega_{\mathrm{CDM}}$ represents the cold matter density of the universe and $h$ parameterises the present Hubble rate, $H_{0}=100 h \mathrm{Km} \mathrm{sec}^{-1} \mathrm{Mpc}^{-1}$, with $h=0.72 \pm 0.08$. On the other hand, extremely accurate numerical packages allow to compute, with a typical accuracy of $1 \%$ or better, the neutralino thermal relic abundance for a given supersymmetric setup [34, 35, 36].

We will require that neutralinos either totally or partly contribute to the inferred dark matter density. We point out that in most models of low energy SUSY it turns out that the computed amount of thermal relic neutralinos is typically larger than what indicated in Eq. (13) above ${ }^{3}$. With this in mind, and allowing for the presence of extra DM components beside neutralinos, we will only consider as a constraint the 95\% C.L. upper bound on the thermal relic abundance of neutralinos derived from Eq.(13):

$$
\Omega_{\tilde{\chi}_{1}} h^{2}<0.129
$$

As regards prospects for direct and indirect detection of neutralino dark matter within the constrained MSSM, 41, 42, 43, it has been shown that the most favorable parameter space points lie in the focus point-hyperbolic branch region. For instance, regarding the muon flux from neutralino pair-annihilation in the center of the Earth or of the Sun, one of the most promising indirect detection strategies, planned neutrino telescopes [43, 44, 45] will probe, within the CMSSM, practically only regions at low neutralino masses and large higgsino content. On the other hand, it has been shown [4] that direct detection spin-dependent searches have typical projected sensitivities lying orders of magnitude far from the expected signal. As regards, finally, direct detection through spin-independent interactions, once again promising signals are predicted only in the low neutralino masses and/or large higgsino fractions parameter space points of the CMSSM. To summarise, at present and future facilities, direct and indirect dark matter searches will compete with

\footnotetext{
${ }^{3}$ It should be noted that in several examples, the presence of extra components in the Universe energy density at neutralino decoupling, for instance a scalar field in a Brans-Dicke-Jordan cosmology 37, a quintessential field [38, 39, 40], or the shear energy density associated to primordial anisotropies [37, only strongly enhances the predicted neutralino abundance: for instance, the presence of a quintessential scalar field may lead to enhancements in the neutralino relic density up to six orders of magnitude [40.
} 
the LHC only in a very limited CMSSM parameter space region, in the focus point strip at moderate values of $M_{1 / 2}$, and therefore at low neutralino masses.

Combining all the constraints given by Eqs.(10, 11, 12) and in particular Eq. (14), apart from the well-known bulk region, at very low soft breaking masses, which will be thoroughly probed by Tevatron and the LHC, three main regions survive in the CMSSM parameter space:

- The Stau Coannihilation Regions: If the lightest stau and the lightest neutralino are quasi degenerate in mass, efficient stau-stau as well as stau-neutralino (co-)annihilations [46] significantly contribute in suppressing the thermal $\chi_{1}$ relic abundance ${ }^{4}$. Most of these regions will be accessible to the LHC, at any value of $\tan \beta$, with the possible exception of very large values of $M_{1 / 2}$.

- Funnel Regions: In these regions, the bino-bino annihilation cross section is greatly enhanced through resonant $s$-channel exchange of the heavy neutral Higgses $A$ and $H$. The conditions required for the resonant enhancement are a large value for $\tan \beta$ and $\mu<0$, which are necessary to fulfill the relation $2 m_{\chi_{1}^{0}} \approx m_{A}$. Here LHC might cover the parameter space corresponding, roughly, to $M_{1 / 2} \leq 1 \mathrm{TeV}$.

- Focus point or Hyperbolic Branch Regions: These regions are narrow zones corresponding to large values of $m_{0}$, yielding a low value for the $\mu$ parameter. The focus point region lies close to where radiative electroweak symmetry breaking (EWSB) is not valid, and is also relatively unstable numerically. The main feature is that a non-negligible higgsino fraction in the lightest neutralino is produced. The LHC reach of this region is pretty limited, owing to the large values of $m_{0}$ and $M_{1 / 2}$, yielding very heavy gluinos and squarks.

The 'smallness' of the surviving regions in the CMSSM is a result of the strict universality assumptions on the soft masses at the high scale within the CMSSM. It has been shown that, relaxing some assumptions about the Higgs [48, 49] or the sfermion [50] soft SUSY breaking mass universality, other coannihilation partners may arise, such as the sneutrino [48, 50] or the bottom squark [50], leading to larger allowed regions in the parameter space compared to the present situation.

We compute the neutralino relic density through the darkSUSY package 34, which merges the accurate numerical SUSY RGE's evolution package ISASUGRA [51] with the state-of-the-art technique to treat the thermal averaging of cross sections, coannihilations, resonances and threshold effects 35. To evaluate the lepton flavour violating rates we use the complete formulæof Ref. [16, in the mass-eigenstate basis.

\footnotetext{
${ }^{4}$ Similar mechanisms with the lightest stop are possible in regions with very large trilinear scalar couplings [35, 47. We do not consider these regions in the present work.
} 


\section{The Canonical $\left(m_{0}-M_{1 / 2}\right)$ Plane and LFV}

We present our results for the effects of LFV on the CMSSMRN parameter space in the $\left(m_{0}-M_{1 / 2}\right)$ plane, as customary in CMSSM studies. We then concentrate on the regions of the parameter space which satisfy all the cosmo-phenomenological constraints outlined in the previous Section, which are plotted as green contours. As a starting point, in this Section we present the results for the 'best case' scenario in the plane $\left(M_{1 / 2}, m_{0}\right)$ for a fixed value of $\tan \beta$ and $A_{0}=0$, and a given sign of $\mu$. We will always show in what follows both a small and a large $\tan \beta$ case, respectively setting $\tan \beta=10$ and 50 (for thourough discussions about the $\tan \beta$ dependence in LFV processes see Ref. [3, 16]), while we devote sec. 3.1 to the question of a non-vanishing trilinear coupling $A_{0}$.

In Fig. 1 we show the isolevel curves of constant $\operatorname{BR}(\tau \rightarrow \mu \gamma)$ for $\tan \beta=10$ and $\mu>0$. From the figure, we see that the present experimental limit already starts probing the region of the parameter space at low $m_{0}$ and $M_{1 / 2}$, which is likely to be tested at Tevatron. The green patch representing the coannihilation region intersects the isolevels corresponding to $\operatorname{BR}(\tau \rightarrow \mu \gamma)$ between $10^{-10}$ and $10^{-11}$, a level of sensitivity likely to be achieved only at dedicated $\tau$-factories. In this low $\tan \beta$ coannihilation region, which will be entirely probed at the LHC, LFV does not significantly impact on the quest for SUSY.

The situation changes however for the large $\tan \beta$ region, where one expects a large

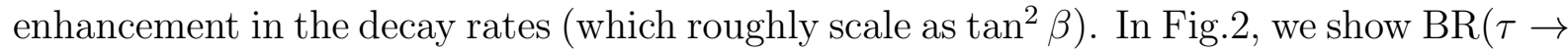
$\mu \gamma)$ iso-contours for $\tan \beta=50$. Notice that we have now chosen $\mu<0$ so that the A-pole funnel regions (see figure caption) also appear within the allowed parameter space. Here we see that even the present experimental bound on $\operatorname{BR}(\tau \rightarrow \mu \gamma)$ can exclude values of $m_{0}$ up to $800 \mathrm{GeV}$ and up to $300 \mathrm{GeV}$ in $M_{1 / 2}$. The intersection of the isolevel curves with the coannihilation region and with the funnel takes place for $\operatorname{BR}(\tau \rightarrow \mu \gamma) \approx 10^{-8}$ and extends beyond $10^{-9}$, well within the reach of present as well as proposed B-factories.

The amplitude of $\mu \rightarrow e \gamma$ is $U_{e 3}$ dependent, as mentioned earlier. In Fig. 3, we plot isolevels corresponding to the present experimental limit on $\mathrm{BR}(\mu \rightarrow e \gamma)$ for various values of $U_{e 3}$. Notice that we use here as a parameter the mixing angle $s_{13}=e^{-i \delta} U_{e 3}$. We choose again $\tan \beta=50$ and $\mu<0$. The results are remarkable. Taking $s_{13}=0.2$, close to the present experimental upper bound from CHOOZ, would rule out the whole parameter space. This fact can be interpreted either as a negative result for a large $U_{e 3}$ or for a large value of $\tan \beta$. In any case, it implies relevant consequences for supersymmetric seesaw models. In the plot, we show values starting from $s_{13}=0.05$. We see that a value of $s_{13}$ ten times smaller than the present bound $\left(s_{13}=0.02\right)$ can still exclude $M_{1 / 2}<750 \mathrm{GeV}$ for any value of $m_{0}$.

Finally, we show in Fig. 4 the isolevel curves corresponding to various values of $\operatorname{BR}(\mu \rightarrow e \gamma)$ in the worst case scenario, which, we recall, features a CKM-like mixing in the neutrino Yukawa couplings. Though $\tan \beta$ is fixed to a large value, 50, LFV rates are nonetheless rather suppressed: the current experimental bound, for instance, does not give any constraint on the SUSY parameter space. On the other hand, even in this case an improvement in the experimental sensitivity on $\operatorname{BR}(\mu \rightarrow e \gamma)$ may lead to access a large part of the cosmo-phenomenologically viable regions. From this point on we will however only consider the best case scenario, because it typically gives more interesting constraints and elucidates the possible relevance of $U_{e 3}$ for LFV. 


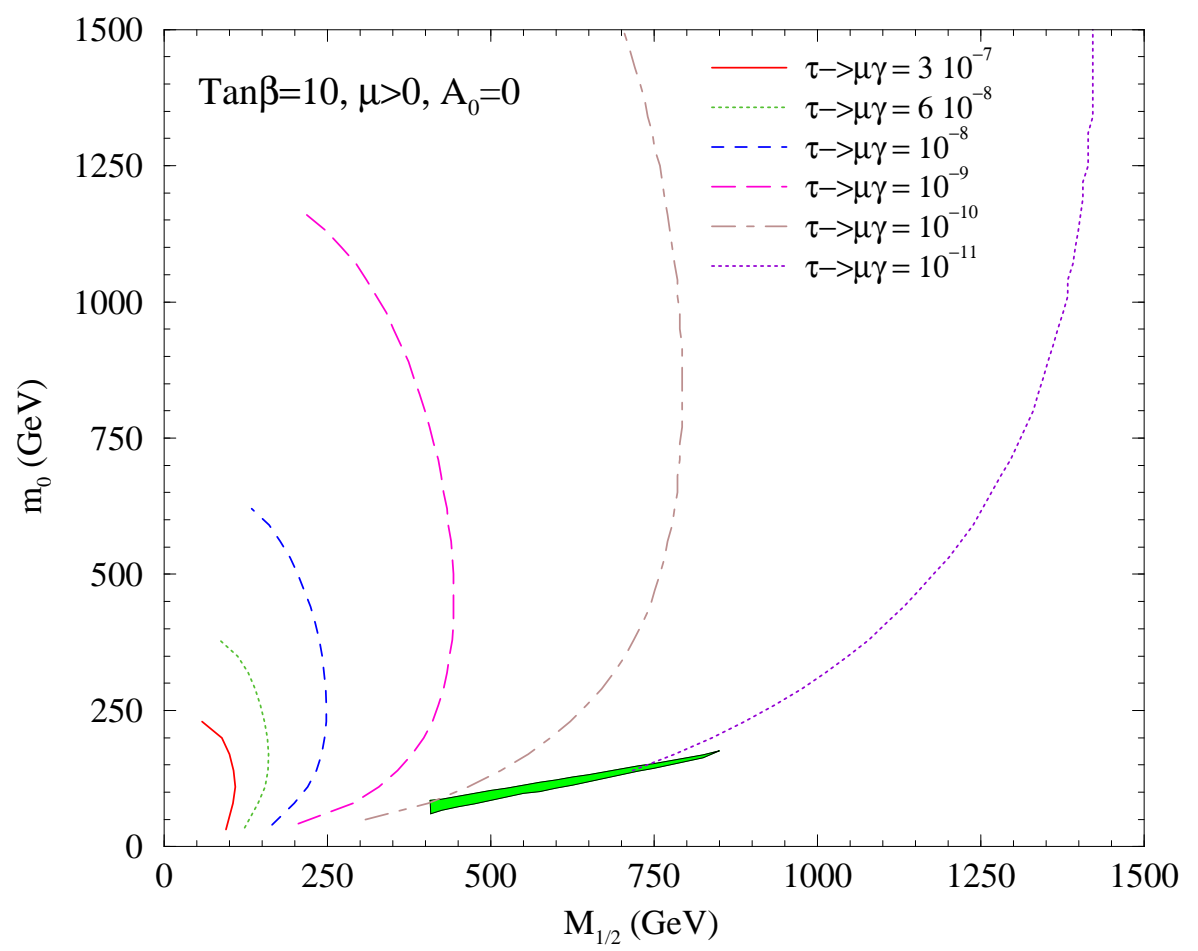

Figure 1: The area shaded in green shows the parameter space, in the $\left(M_{1 / 2}, m_{0}\right)$ plane, allowed by all phenomenological and cosmological constraints, for $\tan \beta=10, \mu>0$ and $A_{0}=0$. The lines correspond to various isolevel curves at $\operatorname{BR}(\tau \rightarrow \mu \gamma)=3 \cdot 10^{-7}, 6 \cdot 10^{-8}$, $10^{-8}, 10^{-9}, 10^{-10}, 10^{-11}$. The starting point of each line is dictated by the condition $m_{\tilde{\chi}_{1}}=m_{\tilde{\tau}}$, which fixes the lowest possible value of $m_{0}$. The isolevel curves terminate where radiative electroweak SUSY breaking (EWSB) conditions cannot be fulfilled, therefore giving the largest possible value of $m_{0}$. 


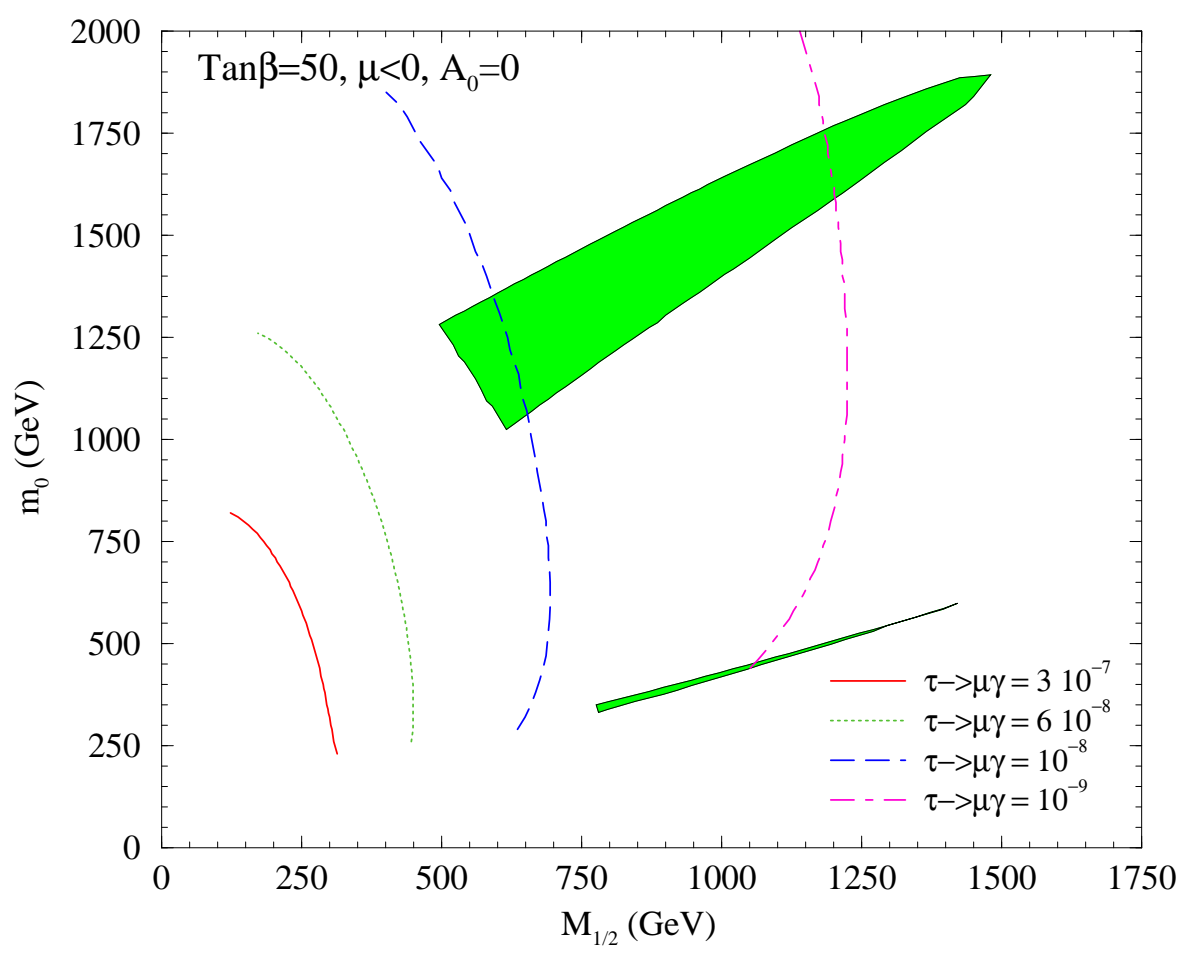

Figure 2: The area shaded in green shows the parameter space, in the $\left(M_{1 / 2}, m_{0}\right)$ plane, allowed by all phenomenological and cosmological constraints, for $\tan \beta=50, \mu<0$ and $A_{0}=0$. The upper area at large $m_{0}$ represents the funnel region. The lines correspond to various isolevel curves at $\operatorname{BR}(\tau \rightarrow \mu \gamma)=3 \cdot 10^{-7}, 6 \cdot 10^{-8}, 10^{-8}, 10^{-9}$. As in Fig. 11, each line starts at $m_{\tilde{\chi}_{1}}=m_{\tilde{\tau}}$ and ends where REWSB conditions are no longer fulfilled. 


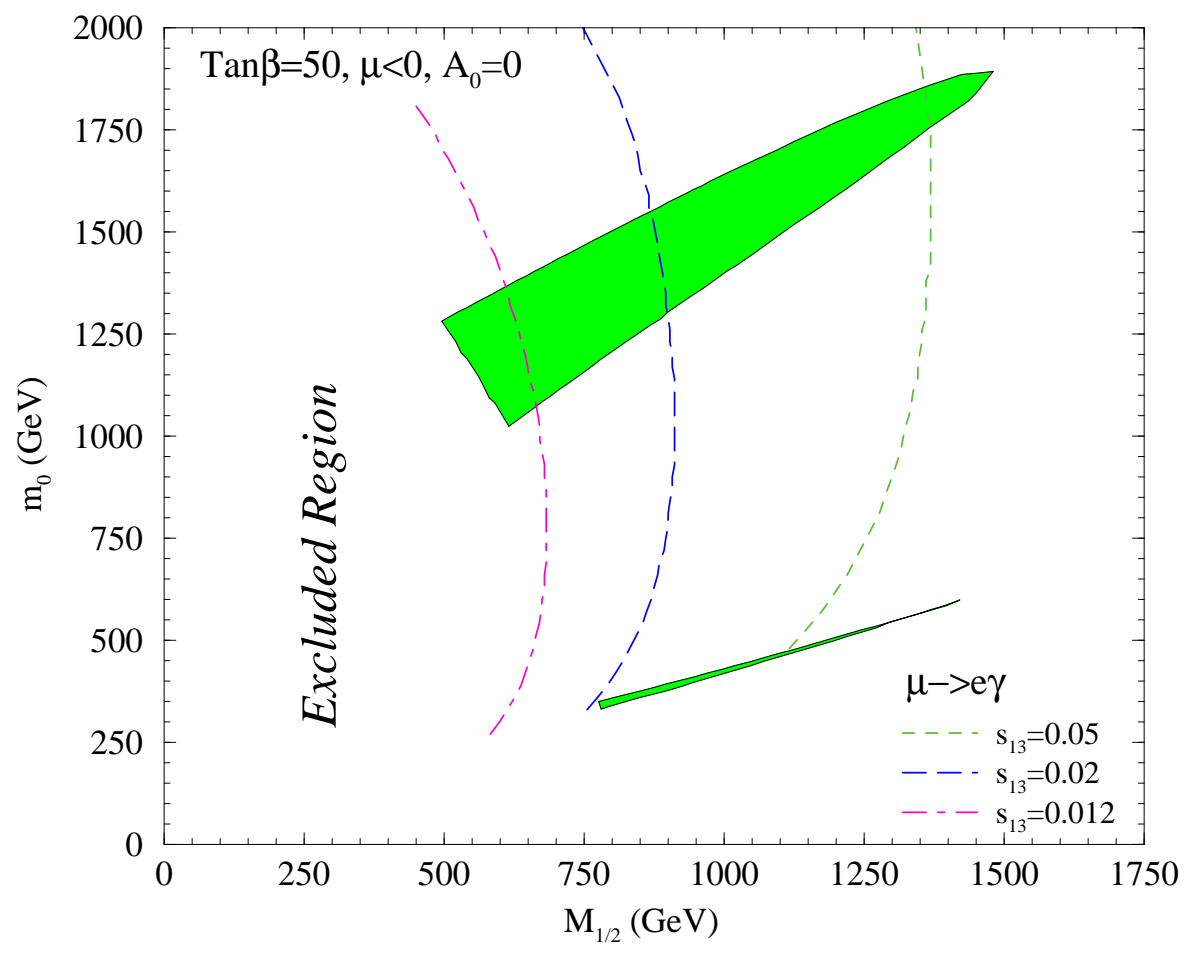

Figure 3: The area shaded in green shows, as in Fig. 2, the allowed parameter space, in the $\left(M_{1 / 2}, m_{0}\right)$ plane, for $\tan \beta=50, \mu<0$ and $A_{0}=0$. The lines correspond this time to the exclusion curves dictated by the current experimental bound on $\operatorname{BR}(\mu \rightarrow$ e $\gamma)$, for various values of $s_{13}=0.05,0.02$ and 0.012: points lying to the left of these curves are henceforth ruled out by the current bound $\operatorname{BR}(\mu \rightarrow e \gamma)<1.2 \cdot 10^{-11}$. The extreme case $s_{13}=0.2$ would rule out the entire parameter space, and is not shown. Again, as in the preceding figures, each line begins at $m_{\tilde{\chi}_{1}}=m_{\tilde{\tau}}$ and ends where EWSB conditions are no longer fulfilled. 


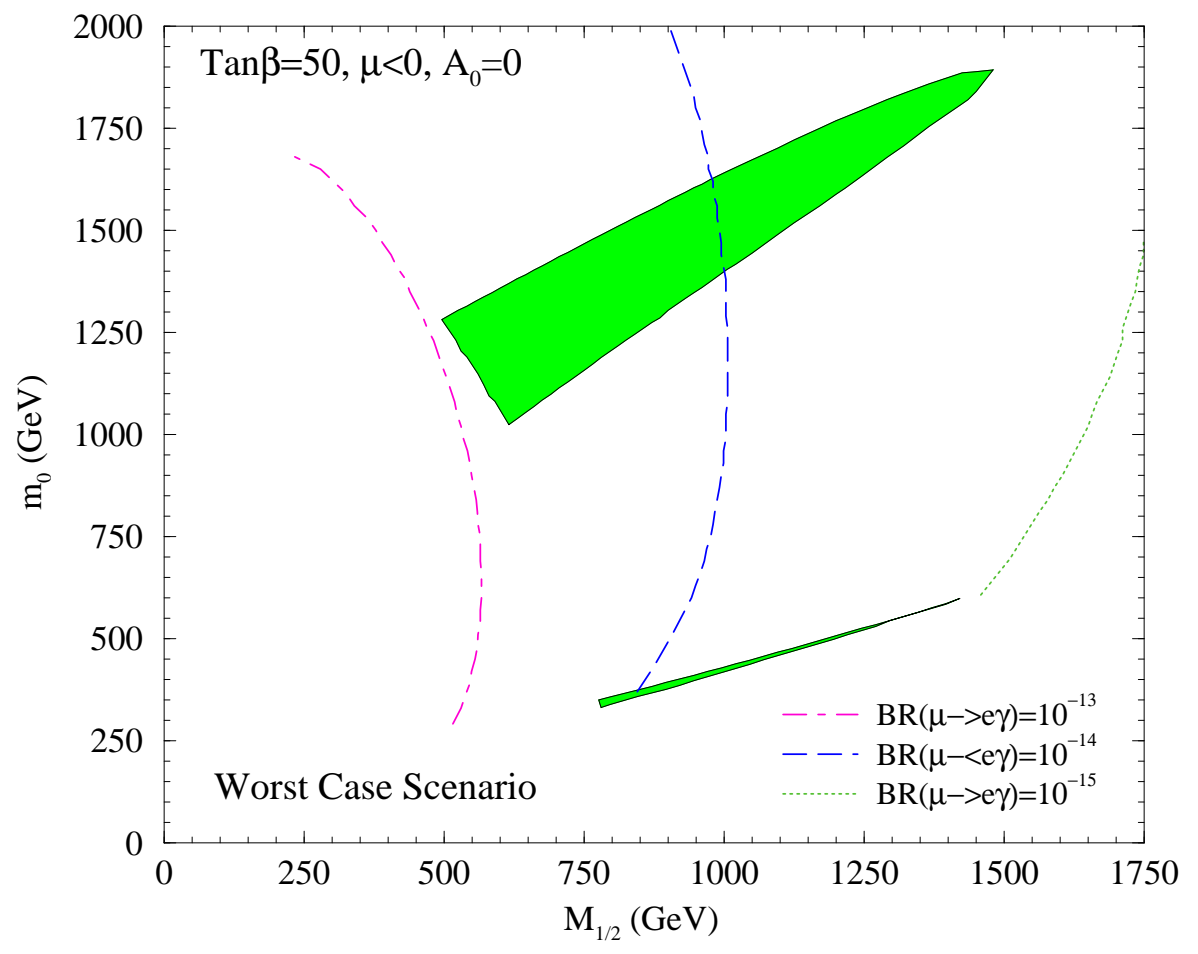

Figure 4: Different isolevel curves of $\mathrm{BR}(\mu \rightarrow e \gamma)$ for the worst case scenario, i.e. corresponding to a CKM-like mixing in the neutrino mixing matrix, on the same parameter space as in Fig. 3, at $\tan \beta=50, \mu<0$ and $A_{0}=0$. 


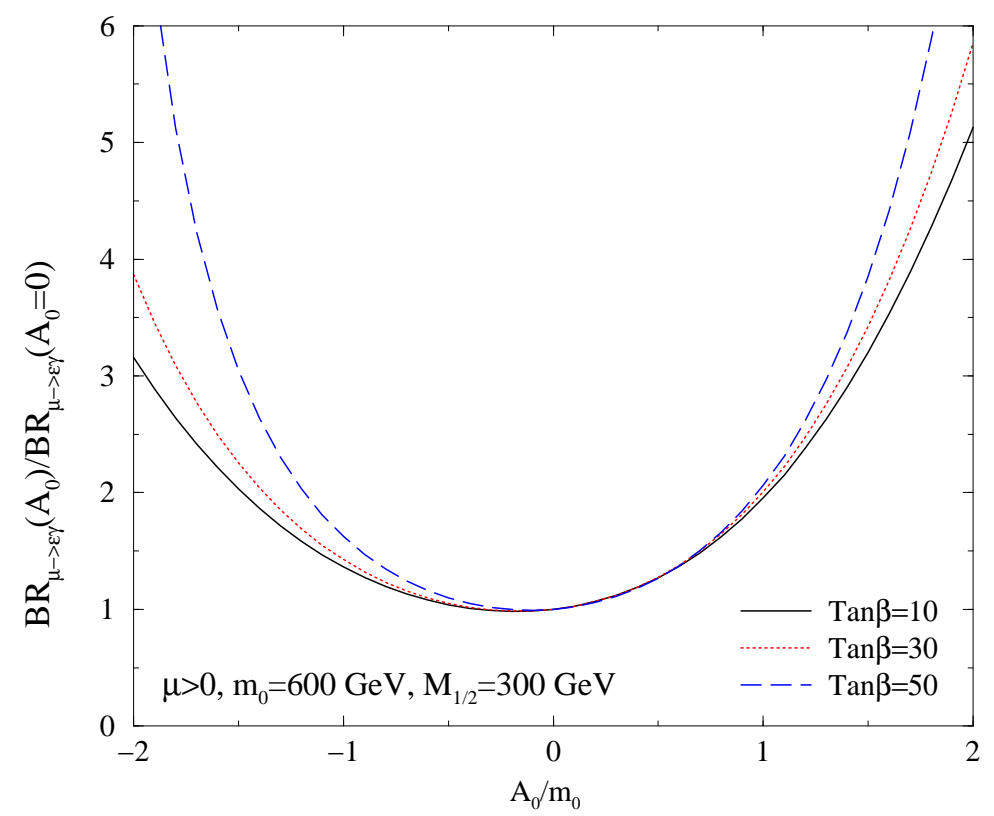

Figure 5: The dependence of $\mathrm{BR}(\mu \rightarrow e \gamma)$ on the trilinear coupling $A_{0}$, for the particular choice $\mu>0, m_{0}=600 \mathrm{GeV}$ and $M_{1 / 2}=300 \mathrm{GeV}$. The three lines correspond to the ratio of $\operatorname{BR}(\mu \rightarrow e \gamma)$ computed at a given $A_{0} \neq 0$ and its value at $A_{0}=0$, for three different values of $\tan \beta=10,30,50$.

\subsection{The Dependence on $A_{0}$}

Before proceeding to the next section, we pause to comment on the implications of a nonzero trilinear coupling on the LFV results. As mentioned above, so far the parameter $A_{0}$ has been set to zero. We argue here that any value $A_{0} \neq 0$ would give a larger branching ratio and therefore the case $A_{0}=0$ provides a lower bound.

A non-zero $A_{0}$ affects the amplitudes of $l_{j} \rightarrow l_{i} \gamma$ decays in three ways: (i) The first effect comes from RG scaling. From Eq.(3), we see that the RG-generated off-diagonal flavour violating mass-insertions are proportional to $A_{0}^{2}$. This is the dominant effect coming from a non-zero $A_{0}$, and it is independent on its sign. (ii) Another way in which $A_{0}$ affects the branchings is through modifications to the particle spectrum. In particular, the parameter $\mu$ is sensitive to the value and to the sign of $A_{0}$. However, this produces only a small effect in the branchings ratios, because $\mu$ in the CMSSM is associated with the heavier chargino and the heaviest neutralino states. This effect is responsible of the manifest, though small, asymmetry for positive and negative values of $A_{0}$ (see Fig. 5). (iii) Last, the left-right mixing elements of the slepton mass matrix contain $A_{0}$ in addition to the $\mu \tan \beta$ factor. This 'left-right' mixing may play an important role in enhancing the decay amplitudes [16, 52]. However, it turns out that for most of the parameter space, the main contribution to this mixing is from the $\mu \tan \beta$ terms rather than from non-zero values of $A_{0}$.

As a quantitative reference, in Fig 5 we show, as a function of $A_{0} / m_{0}$, the ratio between $\operatorname{BR}(\mu \rightarrow e \gamma)$ and its value at $A_{0}=0$, for different values of $\tan \beta$. In agreement with 
what stated above, the minimum value of the branching is obtained for $A_{0}=0$, and there is only a slight dependence with the sign of $A_{0}$. Thus all the predictions presented in this and the later section have actually to be regarded as lower bounds on LFV rates. Let us finally point out that the overall variation induced by a non-zero value of the $A_{0}$ parameter lies within less than one order of magnitude. Finally, we remark that we numerically verified that these conclusions do not depend qualitatively on the particular parameter space point under consideration.

\section{Coannihilations, Funnels and Focus Point}

LFV processes constitute a meaningful constraint on SUSY models, as the results for the 'best case' scenario presented in the preceding section manifestly show. We will demonstrate that sometimes they even do better than direct accelerator SUSY searches. Given this situation, we now come back to the question of the complementarity between the various roads leading to SUSY discovery, directly comparing the LHC reach with the prospects for LFV experiments. We therefore now proceed to detail on the impact of LFV rates for each of the three allowed regions of the CMSSMRN, for benchmark $\tan \beta$ values. Namely, we will concentrate on (a) the coannihilation region, (b) the funnel region and finally (c) the focus point regions, as mentioned at the end of Sec. 3.

\subsection{Coannihilation Strips}

The defining condition for this region is $m_{\tilde{\chi}_{1}} \simeq m_{\tilde{\tau}_{1}}$, where $\tilde{\tau}_{1}$ denotes the lighter stau. In the present section, we stick to the points in the $\left(m_{0}-M_{1 / 2}\right)$ plane which saturate the limiting condition $m_{\tilde{\chi}_{1}}=m_{\tilde{\tau}_{1}}$, maximising the extension of the coannihilation strip. We further choose two values for $\tan \beta, 10$ and 50, setting, in the first case $\mu>0$, while in the second $\mu<0$. The scalar trilinear coupling $A_{0}$ is always set to zero (see Sec 3.1).

In Fig. 6] we show our predictions for $\operatorname{BR}(\tau \rightarrow \mu \gamma)$ and for $\operatorname{BR}(\mu \rightarrow e \gamma)$ in the $\tan \beta=10, \mu>0$ case. The yellow region dictates the lower bound on the neutralino mass, provided by the LEP constraint on the mass of the lightest $C P$-even Higgs boson $m_{h}$ [24. On the other hand, the cyan region gives the upper bound, dictated by the point where $m_{\tilde{\chi}_{1}}=m_{\tilde{\tau}_{1}}$ and $\Omega_{\tilde{\chi}_{1}} h^{2}=0.129$, i.e. the maximal neutralino mass in the coannihilation strip compatible with dark matter constraints [10. We also show the current and projected experimental sensitivity for $\operatorname{BR}(\mu \rightarrow e \gamma)$. We stress that all the parameter space points shown in the plot will be within the expected sensitivity of CERN $L H C$, as the latter extends, for this value of $\tan \beta$, up to approximately $m_{\tilde{\chi}_{1}} \simeq 550 \mathrm{GeV}$ in the coannihilation strip. Clearly, for such a low value of $\tan \beta$, LFV rates are rather suppressed, and at present one can just exclude a narrow region at low neutralino masses provided $s_{13}$ is close to its present upper bound. Moreover, $\operatorname{BR}(\tau \rightarrow \mu \gamma)$ lies at least two orders of magnitudes below the planned experimental sensitivity. Interestingly enough,

in case the experimental sensitivity on $\operatorname{BR}(\mu \rightarrow e \gamma)$ is lowered down to $10^{-14}$, it will be possible to detect, within this scenario, $\mu \rightarrow e \gamma$ for $s_{13}$ as low as $10^{-2}$.

The gray shaded band on the $\operatorname{BR}(\tau \rightarrow \mu \gamma)$ line is obtained by varying the parameters within the coannihilation region. Although the range of $m_{0}$ at a given $M_{1 / 2}$ (and therefore 


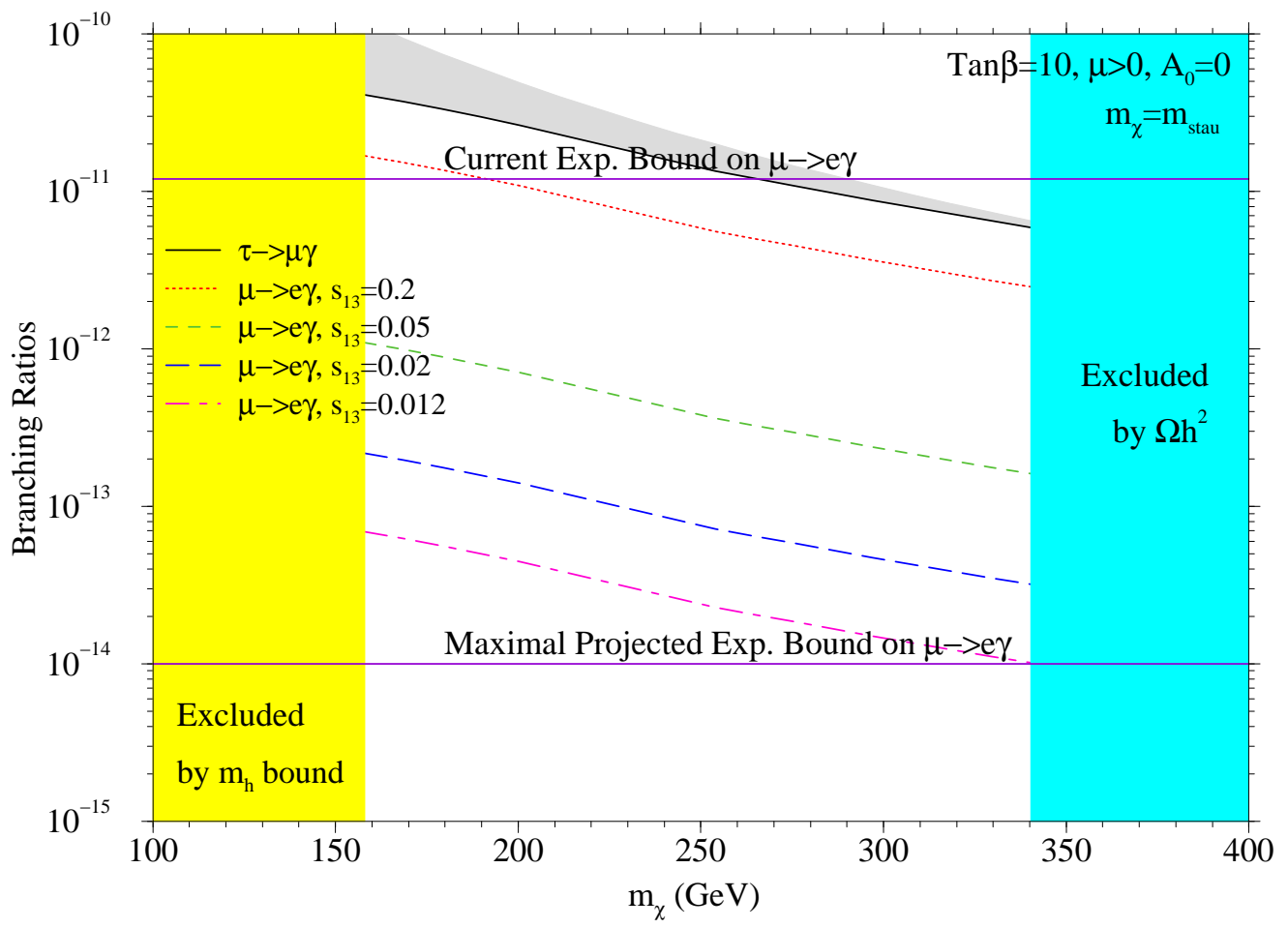

Figure 6: $\mathrm{BR}(\tau \rightarrow \mu \gamma)$ and $\mathrm{BR}(\mu \rightarrow e \gamma)$, for various values of $s_{13}=0.2,0.05,0.02$ and 0.012 , along the line, in the $\left(M_{1 / 2}, m_{0}\right)$ plane for $\tan \beta=10, \mu>0$ and $A_{0}=0$, where $m_{\tilde{\chi}_{1}}=m_{\tilde{\tau}_{1}}$, i.e. in the lowest part of the coannihilation strip. The yellow shaded area at low neutralino masses is ruled out by the LEP bound on $m_{h}$, while points within the cyan shaded region at large neutralino masses give an $\Omega_{\tilde{\chi}_{1}} h^{2}$ value which exceeds the current WMAP constraint on the cold dark matter density. The region shaded in gray indicates the possible values for $\operatorname{BR}(\tau \rightarrow \mu \gamma)$ which one can obtain varying $m_{0}$ within the parameter space showed in Fig. 1. The shape of these shaded regions is analogous for the other lines referring to $\mathrm{BR}(\mu \rightarrow e \gamma)$ 's. We also show the current and projected sensitivities for $\operatorname{BR}(\mu \rightarrow e \gamma)$. We stress that all the points showed in this plot are within the expected CERN LHC reach at an integrated luminosity $\sim 100 \mathrm{fb}^{-1}$. 
neutralino mass) is exceedingly tiny, the shaded area is somewhat large, the reason being that the iso-level curves of LFV rates, as shown in Fig. 1, are approximately parallel to the coannihilation strip.

As regards the large $\tan \beta$ region, we pick the benchmark value $\tan \beta=50$, and choose $\mu<0$ in Fig. $7(a)$, again along the coannihilation strip. This time, since the isolevel curves intersect the coannihilation area almost orthogonally, the overall dependence on the $m_{0}$ spread is completely negligible, and the gray shaded area is vanishingly small (but depicted over the $\tau \rightarrow \mu \gamma$ line). The lower bound on the neutralino mass is dictated by the upper bound on the inclusive $\operatorname{BR}(b \rightarrow s \gamma)$, which for large $\tan \beta$ and negative sign of $\mu$ strongly limits the low mass region of the parameter space. On the other hand, due to stronger couplings in the relevant (co-)annihilation cross sections, the coannihilation strip extends up to rather large neutralino masses. In the present case, the reach of LHC should approximately coincide with the upper bound on the neutralino mass shown in the plot [26. Noticeably, for such a large value of $\tan \beta$, the current experimental upper bound on $\operatorname{BR}(\mu \rightarrow e \gamma)$ happens to put severe constraints on $s_{13}$ : we can for instance qualitatively conclude that if $\tan \beta$ is so large, then $s_{13}$ must be of the order $10^{-2}$ or less. The $s_{13}=0.2$ line turns out, for instance, to be completely excluded by the present experimental bounds. Concerning future improvements on the experimental sensitivity on $\operatorname{BR}(\mu \rightarrow e \gamma)$, we notice that all the lines showed in Fig. $7(a)$ will certainly be within future reach. On the contrary, the situation for $\operatorname{BR}(\tau \rightarrow \mu \gamma)$ is not equally favorable, not even in this large $\tan \beta$ scenario. Yet, $\operatorname{BR}(\tau \rightarrow \mu \gamma)$ of $\mathcal{O}\left(10^{-8}\right)$ would start probing this region.

Lastly, in Fig. [7 (b) we show what would happen switching the sign of $\mu$ to positive values: the lower limit on $m_{\tilde{\chi}_{1}}$ is now given by the lower bound on $\operatorname{BR}(b \rightarrow s \gamma)$, therefore excluding a smaller region, and the coannihilation strip is also slightly enlarged towards larger masses. The predictions for LFV rates are nevertheless not much affected, except for the parameter space which is overall much wider, thus leaving an appealing window, at low masses, where LFV processes are particularly large, and even $\operatorname{BR}(\tau \rightarrow \mu \gamma)$ may lie within planned experimental sensitivities.

\subsection{A-Pole Funnels}

We now proceed in our analysis moving to the $A$-Pole Funnel regions. The defining condition for this region is $2 m_{\chi_{1}} \simeq m_{A}$, and again we show $\left(m_{0}, M_{1 / 2}\right)$ points which saturate the limiting case for which equality holds. In Fig. 8 we consider $\tan \beta=50$ and $\mu<0$. Also in this case, though the parameter space, as shown in Fig. 2 is by far larger than in the coannihilation strip, the spread in the LFV rates is again remarkably narrow, as can be inferred from the gray shaded region surrounding the $\operatorname{BR}(\tau \rightarrow \mu \gamma)$ line. As before, the lower limit on $m_{\tilde{\chi}_{1}}$ is set by the upper bound on the inclusive branching ratio $b \rightarrow s \gamma$. The projected LHC reach only extends up to $m_{\tilde{\chi}_{1}} \lesssim 500 \mathrm{GeV}$, thus leaving a sizable portion of parameter space outside visibility at the future CERN facility. This is the first instance where LFV experiments actually compete with CERN LHC as an additional road to supersymmetry. In fact, values of $s_{13} \lesssim 0.2$ are already ruled out by the current experimental bounds on $\operatorname{BR}(\mu \rightarrow e \gamma)$. Should the experimental reach for this

branching ratio be lowered down to $10^{-13}$, we would be able to detect $\mu \rightarrow$ er signals 


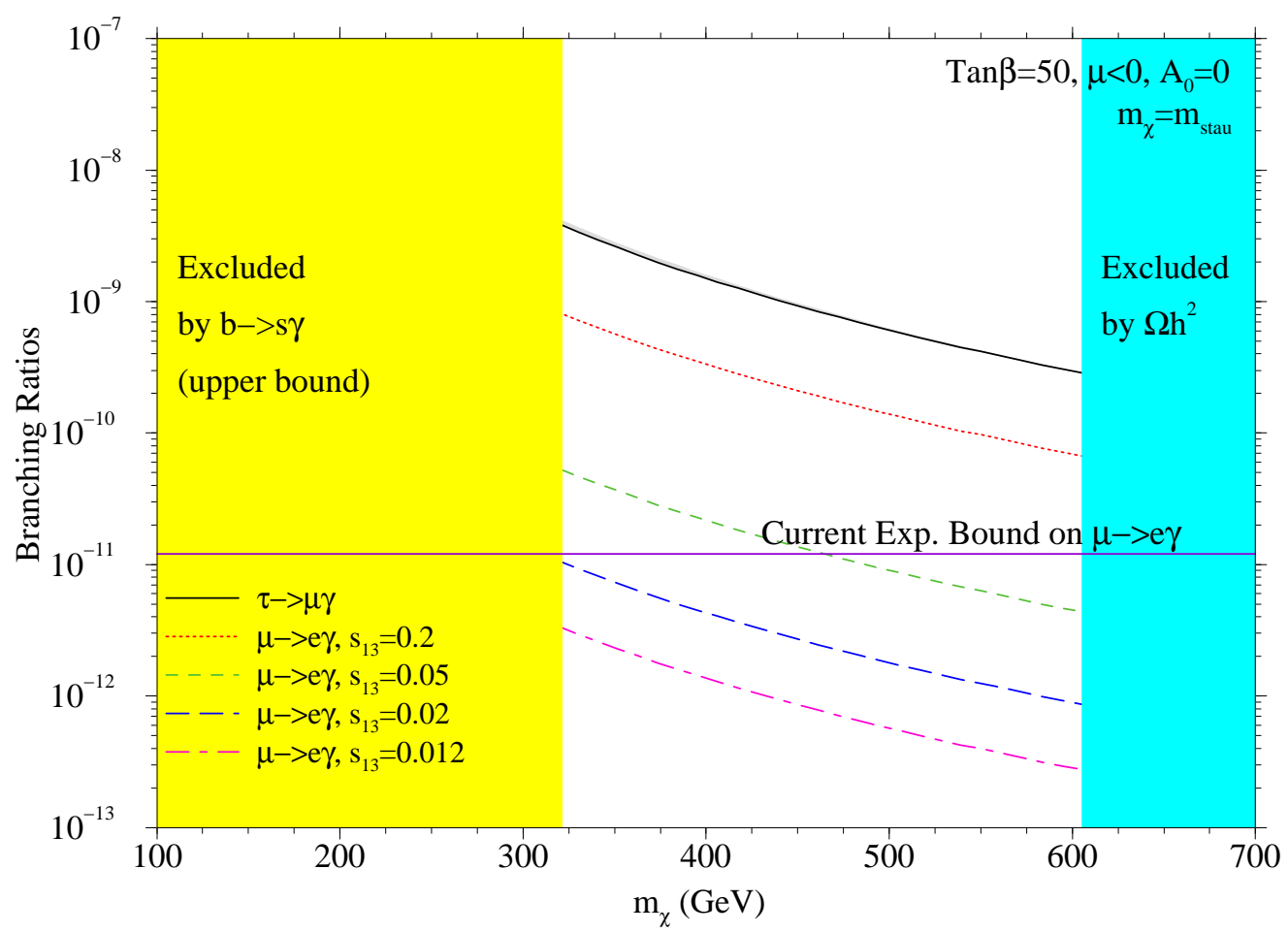

(a)

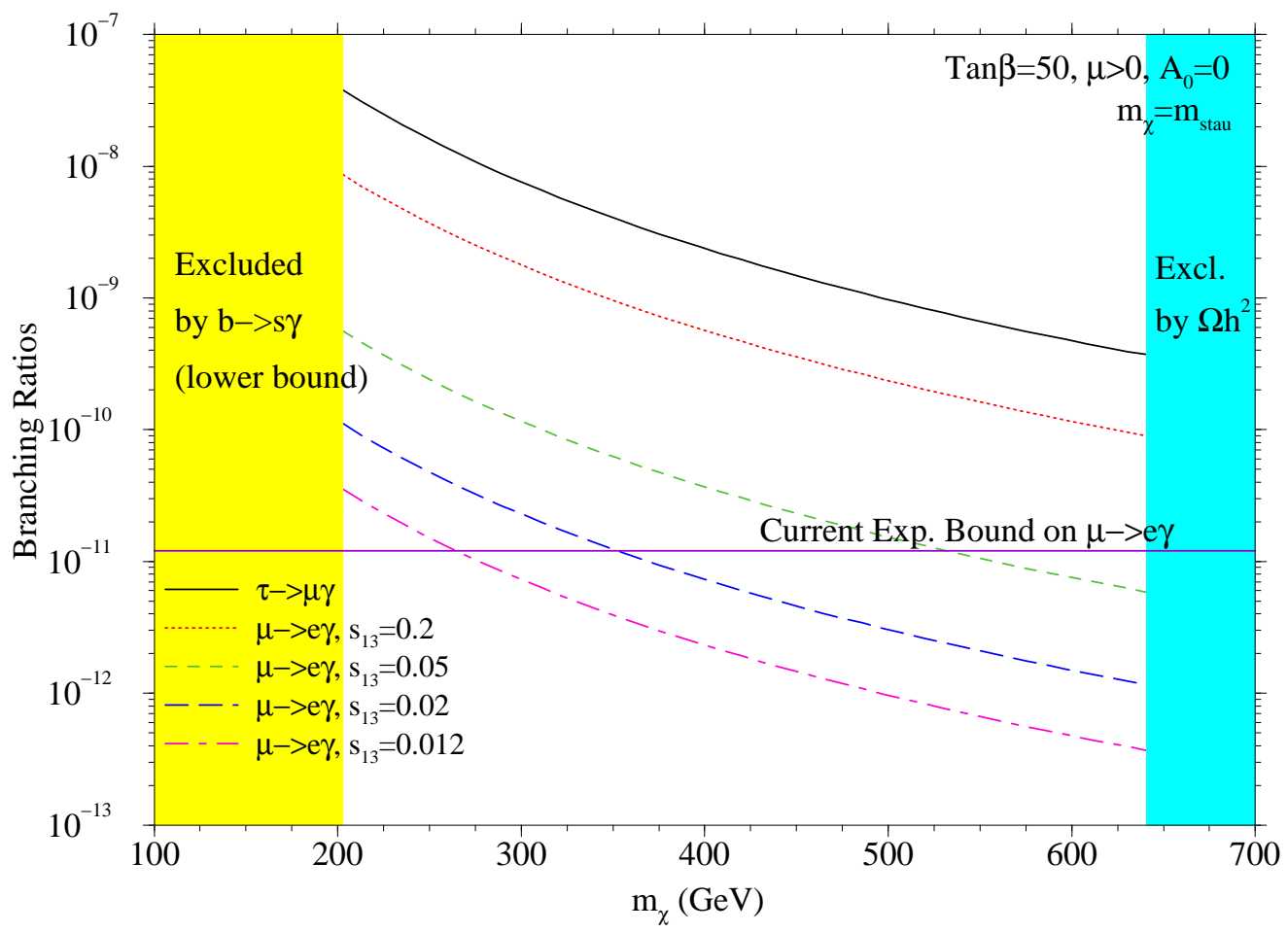

(b)

Figure 7: $(a): \operatorname{BR}(\tau \rightarrow \mu \gamma)$ and $\operatorname{BR}(\mu \rightarrow e \gamma)$, for various values of $s_{13}=0.2,0.05,0.02$ and 0.012 , along the line, in the $\left(M_{1 / 2}, m_{0}\right)$ plane for $\tan \beta=50, \mu<0$ and $A_{0}=0$, where $m_{\tilde{\chi}_{1}}=m_{\tilde{\tau}_{1}}$, i.e. in the lowest part of the coannihilation strip. The yellow shaded area at low neutralino masses is ruled out here by the inclusive $B R(b \rightarrow s \gamma)$ bound, while points within the cyan shaded region at large neutralino masses give $\Omega_{\tilde{\chi}_{1}} h^{2}$ exceeding the current WMAP upper bound on CDM abundance. The current experimental bound on $\mathrm{BR}(\mu \rightarrow e \gamma)$ is shown by an horizontal solid violet line. The region shaded in gray indicates the possible values for $\operatorname{BR}(\tau \rightarrow \mu \gamma)$ which one can obtain varying $m_{0}$ within the coannihilation parameter space showed in the lower areen strip of Fia. I Also here. 


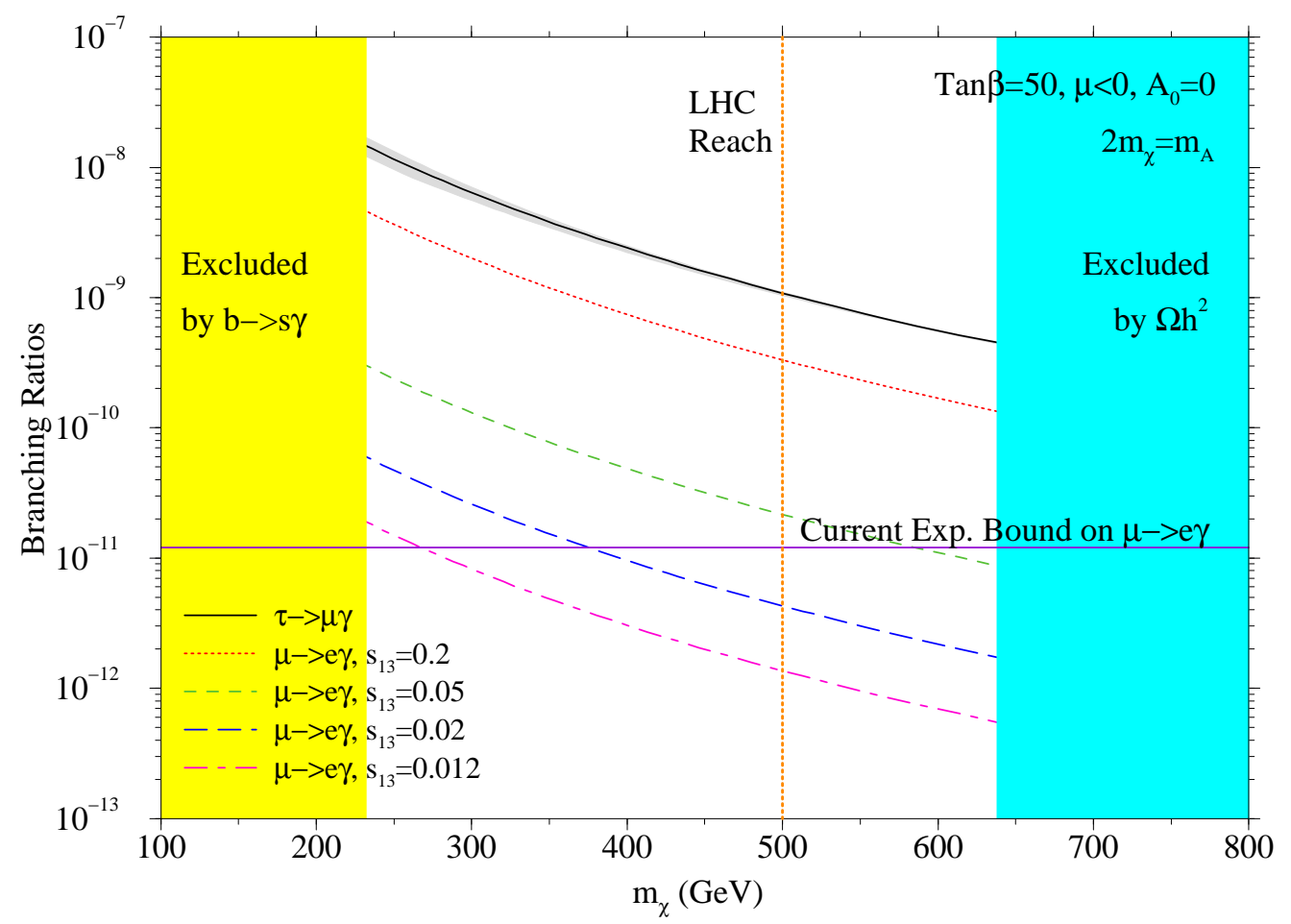

Figure 8: $\operatorname{BR}(\tau \rightarrow \mu \gamma)$ and $\operatorname{BR}(\mu \rightarrow$ e $\gamma)$, for various values of $s_{13}=0.2,0.05,0.02$ and 0.012 , along the line, in the $\left(M_{1 / 2}, m_{0}\right)$ plane for $\tan \beta=50, \mu<0$ and $A_{0}=0$, where $2 \cdot m_{\tilde{\chi}_{1}}=m_{A}$, i.e. in the central part of the funnel region. The yellow shaded area at low neutralino masses is ruled out by the inclusive $B R(b \rightarrow s \gamma)$ bound, while points within the cyan shaded region at large neutralino masses give an $\Omega_{\tilde{\chi}_{1}} h^{2}$ exceeding the current WMAP constraint on neutralino relic density. The region shaded in gray indicates the possible values for $\operatorname{BR}(\tau \rightarrow \mu \gamma)$ which one can obtain varying $m_{0}$ within the funnel parameter space as showed in the upper large green strip of Fig. 圆. We also show the current and projected sensitivities to $\operatorname{BR}(\mu \rightarrow e \gamma)$. The expected CERN LHC reach at an integrated luminosity $\sim 100 \mathrm{fb}^{-1}$ is indicated by the vertical orange dotted line: at neutralino masses larger than $m_{\tilde{\chi}_{1}} \simeq 500 \mathrm{GeV} \mathrm{LHC} \mathrm{will} \mathrm{probably} \mathrm{not} \mathrm{be} \mathrm{able} \mathrm{to} \mathrm{detect}$ supersymmetry in the present parameter space setting. 
in the whole funnel region, provided that $s_{13} \gtrsim \mathcal{O}\left(10^{-2}\right)$. Interestingly, the large mass region lies beyond the expected LHC reach. As in the previous case, we also point out that an experimental sensitivity on $\operatorname{BR}(\tau \rightarrow \mu \gamma)$ of $\mathcal{O}\left(10^{-8}\right)$ would already start probing this region.

\subsection{Focus Point Regions}

In the focus point region very large values of $m_{0}$ lower the Higgs mixing parameter $\mu$, thus entailing the generation of a non-negligible higgsino component in the lightest neutralino ${ }^{5}$. This, in turn, yields an enhancement in the annihilation cross section with respect to the pure bino case, together with coannihilation effects with the next to lightest neutralino and, more importantly, with the lightest chargino, owing to the mass matrix structure of neutralinos and charginos. The combination of coannihilation effects and of a larger annihilation cross section forces the neutralino relic density to drop to very low values, which may be nonetheless compatible with the current dark matter abundance (see the discussion in Sec. 2.1).

The focus point region poses several computational problems, since it lies very close to parameter space points where EWSB fails, and moreover because it is rather fine-tuned, being very sensitive to the input parameters, especially the top mass, $m_{t}$. For this reason, this region of parameter space has sometimes not been included in CMSSM parameter space analyses [48. Nevertheless, we include it in our discussion. Indeed, we consider it useful to analyze the situation from the LFV rates point of view because it will be very hard to probe most of this region at the LHC.

As before, we once again resort to a low (10) and a large (50) value of $\tan \beta$, and we choose to show the extreme part of the focus point region, i.e. that at the largest possible $m_{0}$, and hence where the higgsino content is maximal. Our choice is again motivated by two considerations: first, the region is sufficiently narrow so that LFV rates along the focus point region would appear in any case as single lines; second, choosing the maximal possible higgsino content we extend the parameter space line up to the largest possible neutralino masses.

The lower neutralino mass bound in the focus point region, due to the mass vicinity between the lightest neutralino and chargino, is typically dictated by the chargino mass bound from direct searches. As anticipated, the LHC reach is rather limited in this region: it lies around a neutralino mass of $\sim 200 \mathrm{GeV}$. The large masses characterizing the sfermion spectrum naturally suppress LFV processes, but nonetheless, even in the less favorable case of $\tan \beta=10$, LFV can probe SUSY up to neutralino masses around 800 $\mathrm{GeV}$, corresponding to very large values of the soft breaking masses at the GUT scale, namely $M_{1 / 2} \approx 5.5 \mathrm{TeV}$ and $m_{0} \approx 17.5 \mathrm{TeV}$. As regards the large $\tan \beta$ case, $\operatorname{BR}(\mu \rightarrow e \gamma)$ may be within future experimental reach for neutralino masses in the multi-TeV range, provided $s_{13}$ is of $\mathcal{O}\left(10^{-1} \div 10^{-2}\right)$. Therefore, the focus point region, as well as, in general, the case of a higgsino dominated lightest neutralino, typically tends to favor $\operatorname{BR}(\mu \rightarrow e \gamma)$

\footnotetext{
${ }^{5}$ It should be noted that this situation takes naturally place in several other soft SUSY breaking scenarios, for instance with non-universal gaugino masses [53], where the lightest neutralino is mainly a higgsino; relevant consequences for LFV can be found in Ref. [54].
} 


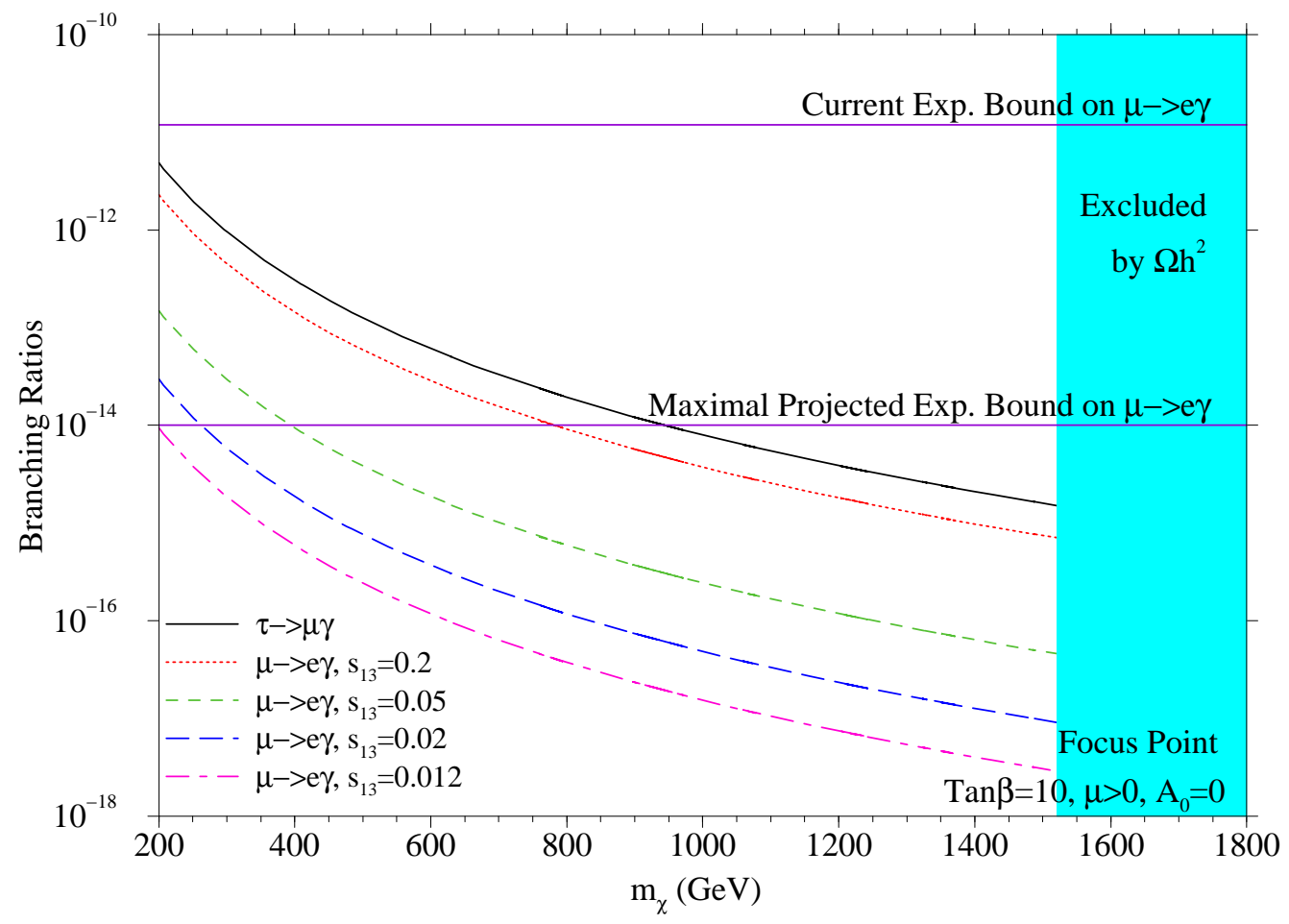

Figure 9: $\operatorname{BR}(\tau \rightarrow \mu \gamma)$ and $\operatorname{BR}(\mu \rightarrow e \gamma)$, for various values of $s_{13}=0.2,0.05,0.02$ and 0.012, along the extreme focus point region, in the $\left(M_{1 / 2}, m_{0}\right)$ plane for $\tan \beta=50, \mu<0$ and $A_{0}=0$. The parameter space points we use here are those such that the higgsino content of the lightest neutralino is maximal. The cyan shaded region at large neutralino masses gives an $\Omega_{\tilde{\chi}_{1}} h^{2}$ exceeding the current WMAP constraint on CDM density. We also show the current and projected sensitivities to $\mathrm{BR}(\mu \rightarrow e \gamma)$. The CERN LHC reach lies at neutralino masses smaller than $200 \mathrm{GeV}$. All the SUSY parameter space points in this plot are therefore outside CERN LHC reach at an integrated luminosity $\sim 100 \mathrm{fb}^{-1}$. 
with respect to direct accelerator searches in the quest for supersymmetry ${ }^{6}$.

\section{The role of $U_{e 3}$}

The importance of the parameter $U_{e 3}$ in its connection with the neutrino mixing matrix and lepton flavour violation in the SUSY seesaw is apparent from the discussions we carried out in the previous two sections. The precise value of $U_{e 3}$ turns out to be of critical importance in at least two contexts in the present analysis:

\section{(i) LFV versus LHC}

Within the coannihilation regions, as we have seen, LFV will play only a "supporting" rôle to the more powerful LHC searches. The first instance where LFV may reveal itself as a superior tool arises in the heavy mass A-pole funnel region. However, the prominence of LFV searches is crucially dependent on $U_{e 3}$. To make this more precise, we plot in Fig. 11 the $\operatorname{BR}(\mu \rightarrow e \gamma)$ as a function of $s_{13}$, showing iso-neutralino mass curves. From the plot it is evident that an experimental sensitivity of $10^{-13}$ would allow to detect LFV as long as $s_{13} \gtrsim 5 \cdot 10^{-3}$. On the other hand, there is a large band, at heavy SUSY particles masses, lying beyond LHC reach, which will be fully accessible to LFV experiments. The same holds true in the focus point region, where, provided $U_{e 3}$ is not too small, $\mathrm{BR}(\mu \rightarrow e \gamma)$ will probe SUSY far more effectively than the LHC.

(ii) $\mu \rightarrow e \gamma$ versus $\tau \rightarrow \mu \gamma$

We have seen that, if $s_{13}$ is not too small, the constraints coming from $\mu \rightarrow e \gamma$ are stronger than those derived from $\tau \rightarrow \mu \gamma$. Hence, there should be a critical value of $s_{13}$ below which $\tau \rightarrow \mu \gamma$ becomes more relevant than $\mu \rightarrow e \gamma$. What is exactly this value? To answer this question we show in Fig. $12 \operatorname{BR}(\mu \rightarrow e \gamma)$ in units of $10^{-13}$ as a function of $s_{13}$, for the particular point at $m_{0}=343, M_{1 / 2}=500, \tan \beta=50$ and $\mu>0$. The dashed-dotted line denotes the value of $\operatorname{BR}(\tau \rightarrow \mu \gamma)$ in units of $10^{-8}$. We observe that the intersection between the $\tau \rightarrow \mu \gamma$ line - which is almost independent of $s_{13}$ - and the $\mu \rightarrow e \gamma$ line takes place at a value of $s_{13}$ close to $10^{-3}$. If $s_{13} \gtrsim 10^{-3}$ then $\mu \rightarrow e \gamma$ is more likely to be observed in future experiments than $\tau \rightarrow \mu \gamma$. Although these results were obtained for a specific value of $m_{0}$ and $M_{1 / 2}$, changes in these parameters are expected to affect equally both processes so that the critical value $s_{13} \approx 10^{-3}$ does not depend on $m_{0}$ or $M_{1 / 2}$ (We have also checked numerically that this is indeed the case). When $s_{13}$ is very small, the contribution to $\operatorname{BR}(\mu \rightarrow e \gamma)$, proportional to the second Yukawa coupling dominates over that proportional to the top Yukawa coupling. In same figure, we show the predictions for $\operatorname{BR}(\mu \rightarrow e \gamma)$ with and without taking into account the effect of the second Yukawa coupling, which we set equal to the charm Yukawa [6]. Notice that such effect is only relevant for $s_{13} \lesssim 7 \times 10^{-4}$.

\footnotetext{
${ }^{6}$ In a recent paper [55], it has been pointed out that at future $e^{+} e^{-}$Linear Colliders with center of mass energy $\sqrt{s} \approx 0.5 \div 1 \mathrm{TeV}$, the accelerator reach in the CMSSM focus point region may be by far larger than that at the CERN LHC
} 


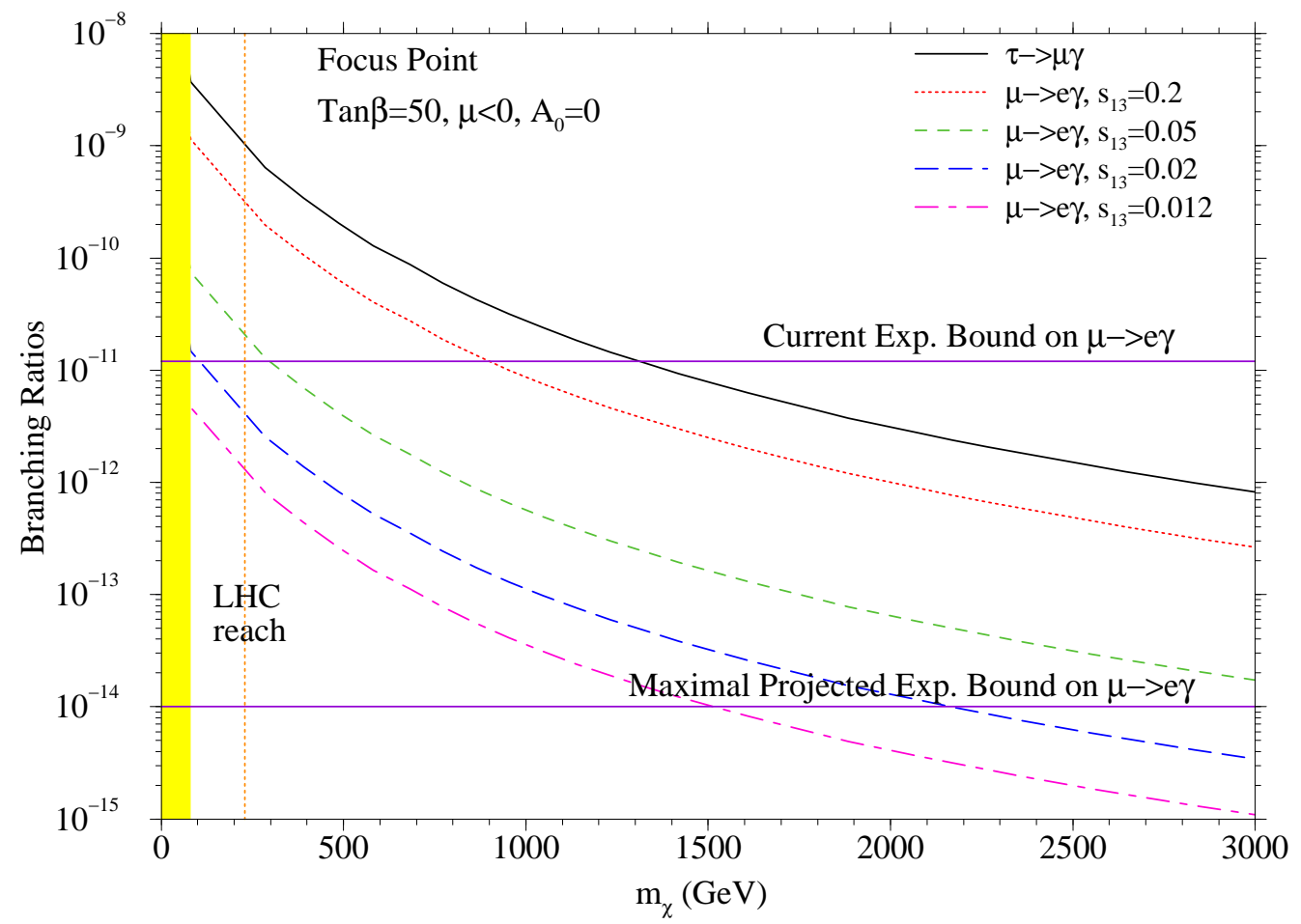

Figure 10: $\operatorname{BR}(\tau \rightarrow \mu \gamma)$ and $\operatorname{BR}(\mu \rightarrow e \gamma)$, for various values of $s_{13}=0.2,0.05,0.02$ and 0.012, along the extreme focus point region, in the $\left(M_{1 / 2}, m_{0}\right)$ plane for $\tan \beta=50$, $\mu<0$ and $A_{0}=0$. Again, the parameter space points we use here are those such that the higgsino content of the lightest neutralino is maximal. We show neutralino masses up to $3 \mathrm{TeV}$, which are still allowed by relic density considerations. The expected sensitivity of CERN LHC is showed by the vertical orange dotted line, while the yellow shaded area on the left indicates the bound stemming from the chargino mass limit set by LEP direct searches. 


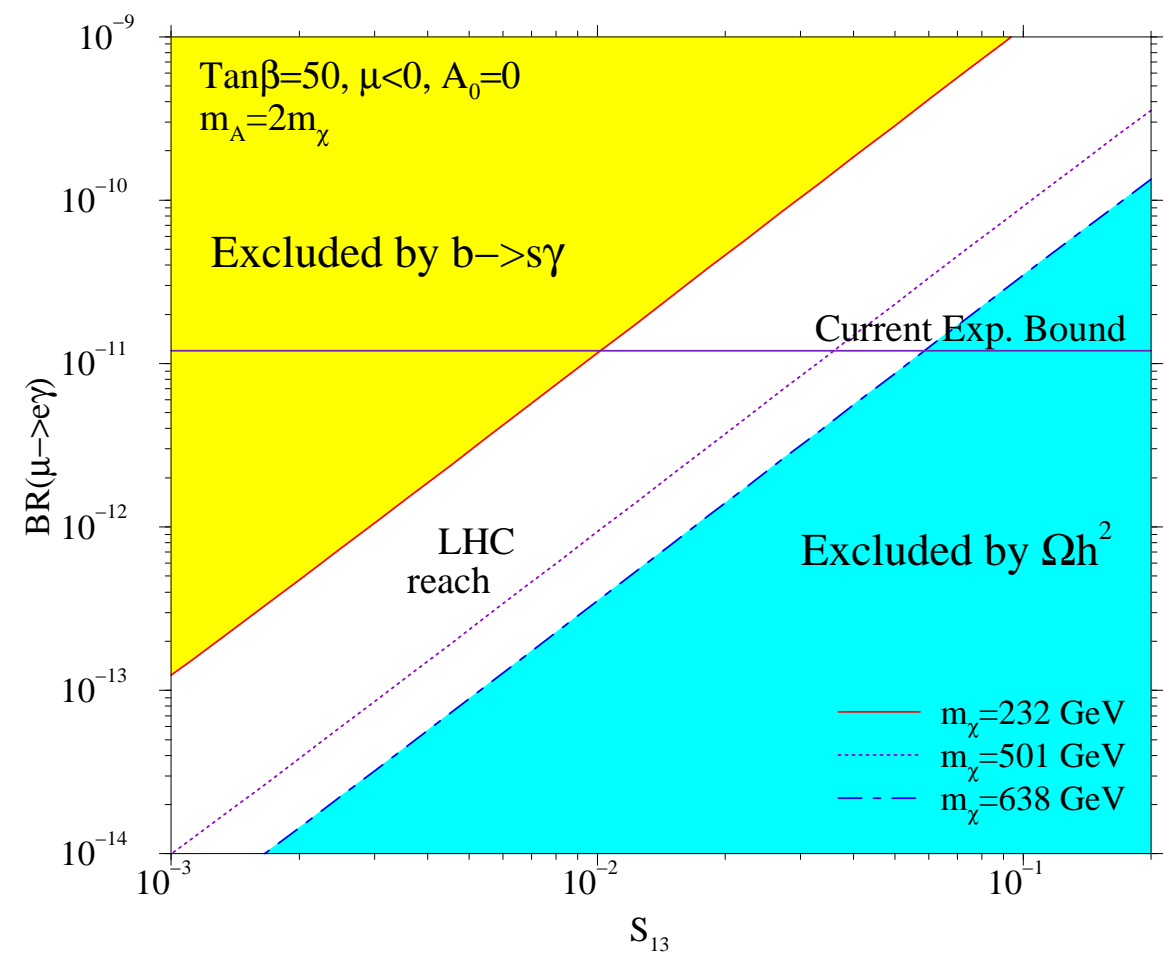

Figure 11: The dependence of $\operatorname{BR}(\mu \rightarrow$ e $\gamma)$ on $s_{13}$, for $\tan \beta=50, \mu<0$ and $A_{0}=0$ along the parameter space line, in the $\left(M_{1 / 2}, m_{0}\right)$ plane corresponding to $2 \cdot m_{\tilde{\chi}_{1}}=m_{A}$, i.e. in the central part of the funnel region. The upper region, shaded in yellow, is ruled out by the $b \rightarrow s \gamma$ bound, while the lower region, shaded in cyan, is disallowed by the $\Omega_{\tilde{\chi}_{1}} h^{2}$ bound on the neutralino relic density. The three lines respectively correspond to the lower and the upper neutralino mass limits and to the largest neutralino mass within LHC reach. We also report the current experimental upper bound on $\operatorname{BR}(\mu \rightarrow e \gamma)$. 


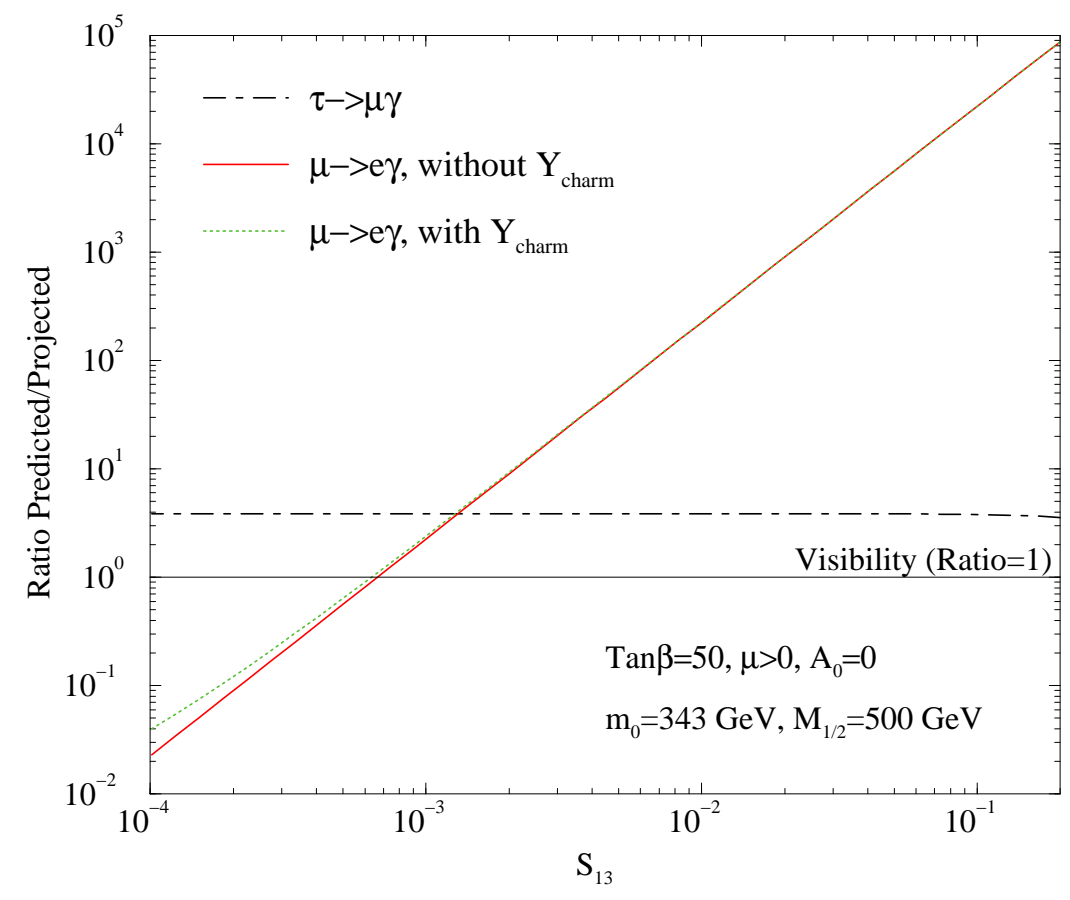

Figure 12: The ratio of the predicted $\operatorname{BR}(\tau \rightarrow \mu \gamma)$ (respectively $\operatorname{BR}(\mu \rightarrow$ e $\gamma)$ ) and the approximate projected maximal sensitivity of $10^{-8}$ (resp. $\left.10^{-13}\right)$ at a particular parameter space point along the coannihilation strip at $\tan \beta=50$, vanishing $A_{0}$ and positive $\mu$. The green dotted line corresponds to the case where the effect of a second non-zero neutralino Yukawa coupling, set equal to the charm quark Yukawa coupling, is taken into account. 


\section{Summary of the Results}

Here we summarise our results on the complementarity of the three search roads for the three allowed regions of the CMSSMRN in the 'best case' scenario.

- Coannihilation Regions: In these regions, which are mostly accessible at LHC, an improvement of two orders of magnitude in the branching ratio sensitivity would make $\mu \rightarrow e \gamma$ visible for most of the parameter space as long as $s_{13} \gtrsim 0.02$, even for the low $\tan \beta$ region. For large $\tan \beta$, independent of $s_{13}, \tau \rightarrow \mu \gamma$ will start probing this region provided a sensitivity of $\mathcal{O}\left(10^{-8}\right)$ is reached.

- A-pole funnel Regions: In these regions the LHC reach is not complete and LFV may be competitive. If $s_{13} \gtrsim 10^{-2}$, the future $\mu \rightarrow e \gamma$ experiments will probe most of the parameter space regions. As before, $\tau \rightarrow \mu \gamma$ will probe this region once the BR sensitivity reaches $\mathcal{O}\left(10^{-8}\right)$.

- Focus Point Regions: Since the LHC reach in this region is rather limited due to the large $m_{0}$ and $M_{1 / 2}$ values, LFV could constitute a privileged road towards SUSY discovery. As we pointed out in Sec. 2. DM searches will also have in future partial access to this region, leading to a new complementarity between LFV and the quest for the cold dark matter constituent of the universe.

\section{Outlook}

So far, the three roads towards SUSY we mentioned are (a) direct accelerator SUSY particles searches; (b) indirect searches through rare $\mathrm{FCNC} / \mathrm{CP}$ violating processes and (c) direct and indirect DM searches. In this decade, these three ways are going to remain the best tools to get evidence for SUSY with foreseeable impressive improvements on their sensitivity reach (in particular, for (a), with the advent of LHC). Our work tries to provide a critical assessment on their complementarity, focusing on the maximal reach of each of them in various viable parameter space regions. To make such an analysis quantitative, one needs a specific low-energy realisation. We chose to work in the context of the CMSSMRN model, because (i) 'CMSSM' is the prototype of 'safe' flavour blind low-energy SUSY extensions to the SM compatible with all the precision SM tests and (ii) 'RN' provides an appealing mechanism to generate neutrino masses. This model is severely constrained by direct SUSY particles searches, FCNC constraints, and, even more strongly, by the information we gained about the amount of CDM from WMAP and large scale structure analysis. As a matter of fact, only three regions of the CMSSMRN parameter space still survive. In the most favorable ('best') case, LFV is certainly complementary to LHC searches in all these three regions and, interestingly, it can probe portions of the parameter space which will be unaccessible to the LHC.

\section{Acknowledgments}

AM and SV acknowledge support from the RTN European project "Physics Across the Present Energy Frontier" HPRN-CT-2000-0148 and from the "Italian University and Re- 
search Ministry" under the program "PRIN:Astroparticle Physics" 2002.

\section{References}

[1] F. Borzumati and A. Masiero, Phys. Rev. Lett. 57, 961 (1986); G. K. Leontaris, K. Tamvakis and J. D. Vergados, Phys. Lett. B171, 412 (1986).

[2] J. A. Casas and A. Ibarra, Nucl. Phys. B 618 (2001) 171 arXiv:hep-ph/0103065.

[3] J. Hisano, D. Nomura and T. Yanagida, Phys. Lett. B 437, 351 (1998) arXiv:hep-ph/9711348; S. F. King and M. Oliveira, Phys. Rev. D 60, 035003 (1999) arXiv:hep-ph/9804283; J. R. Ellis, M. E. Gomez, G. K. Leontaris, S. Lola and D. V. Nanopoulos, Eur. Phys. J. C 14, 319 (2000) arXiv:hep-ph/9911459; S. Baek, T. Goto, Y. Okada and K. i. Okumura, Phys. Rev. D 63, 051701 (2001) arXiv:hep-ph/0002141; J. Hisano and K. Tobe, Phys. Lett. B 510, 197 (2001) arXiv:hep-ph/0102315; D. F. Carvalho, J. R. Ellis, M. E. Gomez and S. Lola, Phys. Lett. B 515, 323 (2001) arXiv:hep-ph/0103256; S. Baek, T. Goto, Y. Okada and K. i. Okumura, Phys. Rev. D 64, 095001 (2001) arXiv:hep-ph/0104146; R. Gonzalez Felipe and F. R. Joaquim, JHEP 0109, 015 (2001) arXiv:hep-ph/0106226; A. Romanino and A. Strumia, Nucl. Phys. B 622, 73 (2002) arXiv:hep-ph/0108275; A. Kageyama, S. Kaneko, N. Shimoyama and M. Tanimoto, Phys. Lett. B 527, 206 (2002) arXiv:hep-ph/0110283 ; A. Kageyama, S. Kaneko, N. Shimoyama and M. Tanimoto, Phys. Rev. D 65, 096010 (2002) arXiv:hep-ph/0112359; F. Deppisch, H. Paes, A. Redelbach, R. Ruckl and Y. Shimizu, Eur. Phys. J. C 28, 365 (2003) arXiv:hep-ph/0206122; D. Falcone, Mod. Phys. Lett. A 17, 2467 (2002) arXiv:hep-ph/0207308; K. S. Babu, B. Dutta and R. N. Mohapatra, Phys. Rev. D 67, 076006 (2003) arXiv:hep-ph/0211068; K. Hamaguchi, M. Kakizaki and M. Yamaguchi, Phys. Rev. D 68, 056007 (2003) arXiv:hep-ph/0212172; J. Hisano and Y. Shimizu, Phys. Lett. B 565, 183 (2003) arXiv:hep-ph/0303071 ; C. S. Huang, T. j. Li and W. Liao, Nucl. Phys. B 673, 331 (2003) arXiv:hep-ph/0304130; T. Fukuyama, T. Kikuchi and N. Okada, Phys. Rev. D 68, 033012 (2003) arXiv:hep-ph/0304190; B. Dutta and R. N. Mohapatra, Phys. Rev. D 68, 056006 (2003) arXiv:hep-ph/0305059; T. F. Feng, T. Huang, X. Q. Li, X. M. Zhang and S. M. Zhao, Phys. Rev. D 68, 016004 (2003) arXiv:hep-ph/0305290; S. F. King and I. N. R. Peddie, Nucl. Phys. B 678, 339 (2004) arXiv:hep-ph/0307091; J. I. Illana and M. Masip, arXiv:hep-ph/0307393 K. S. Babu, T. Enkhbat and I. Gogoladze, Nucl. Phys. B 678, 233 (2004) arXiv:hep-ph/0308093; K. Tobe, J. D. Wells and T. Yanagida, arXiv:hep-ph/0310148, A. Ibarra and G. G. Ross, arXiv:hep-ph/0312138.

[4] J. Sato, K. Tobe and T. Yanagida, Phys. Lett. B 498, 189 (2001) arXiv:hep-ph/0010348.

[5] J. Sato and K. Tobe, Phys. Rev. D 63, 116010 (2001) arXiv:hep-ph/0012333 . X. J. Bi, Eur. Phys. J. C 27, 399 (2003) arXiv:hep-ph/0211236; J. R. Ellis, M. Raidal and T. Yanagida, arXiv:hep-ph/0303242, S. M. Barr, Phys. Lett. B 578, 394 (2004) arXiv:hep-ph/0307372.

[6] A. Masiero, S. K. Vempati and O. Vives, Nucl. Phys. B 649, 189 (2003) arXiv:hep-ph/0209303.

[7] S. Davidson and A. Ibarra, JHEP 0109, 013 (2001) arXiv:hep-ph/0104076; S. Lavignac, I. Masina and C. A. Savoy, Phys. Lett. B 520, 269 (2001) arXiv:hep-ph/0106245; 
J. R. Ellis, J. Hisano, M. Raidal and Y. Shimizu, Phys. Rev. D 66, 115013 (2002) arXiv:hep-ph/0206110. S. Pascoli, S. T. Petcov and W. Rodejohann, Phys. Rev. D 68, 093007 (2003) arXiv:hep-ph/0302054; S. Davidson, JHEP 0303, 037 (2003) arXiv:hep-ph/0302075.

[8] S. T. Petcov, S. Profumo, Y. Takanishi and C. E. Yaguna, Nucl. Phys. B 676, 453 (2004) arXiv:hep-ph/0306195.

[9] S. Pascoli, S. T. Petcov and C. E. Yaguna, Phys. Lett. B 564, 241 (2003) arXiv:hep-ph/0301095.

[10] D. N. Spergel et al., Astrophys. J. Suppl. 148 (2003) 175 arXiv:astro-ph/0302209.

[11] B. A. Campbell, D. W. Maybury and B. Murakami, arXiv:hep-ph/0311244.

[12] T. Blazek and S. F. King, Nucl. Phys. B 662, 359 (2003) arXiv:hep-ph/0211368.

[13] T. Yanagida in Proc. Workshop on Unified Theories EGc., eds. O. Sawada and A. Sugamoto (Tsukuba, 1979); M. Gell-Mann, P. Ramond, and R. Slansky, in Supergravity, eds. D. Freedman et al., (North Holland 1980 Amsterdam); S.L. Glashow, in Quarks and Leptons, Cargèse 1979, eds. M. Lévy, et al., (Plenum 1980 New York), p. 707; R. N. Mohapatra and G. Senjanovic, Phys. Rev. Lett. 44, 912 (1980).

[14] Some recent reviews are: G. Altarelli and F. Feruglio, arXiv:hep-ph/0206077; arXiv:hep-ph/0306265 I. Masina, Int. J. Mod. Phys. A 16, 5101 (2001) arXiv:hep-ph/0107220; R. N. Mohapatra, arXiv:hep-ph/0211252 arXiv:hep-ph/0306016 S. F. King, arXiv:hep-ph/0310204 A. Y. Smirnov, arXiv:hep-ph/0311259

[15] T. Moroi, JHEP 0003, 019 (2000) arXiv:hep-ph/0002208; T. Moroi, Phys. Lett. B 493, 366 (2000) arXiv:hep-ph/0007328; N. Akama, Y. Kiyo, S. Komine and T. Moroi, Phys. Rev. D 64, 095012 (2001) arXiv:hep-ph/0104263; D. Chang, A. Masiero and H. Murayama, Phys. Rev. D 67, 075013 (2003) arXiv:hep-ph/0205111.

[16] J. Hisano, T. Moroi, K. Tobe, M. Yamaguchi and T. Yanagida, Phys. Lett. B 357 (1995) 579 arXiv:hep-ph/9501407; J. Hisano and D. Nomura, Phys. Rev. D 59, 116005 (1999) arXiv:hep-ph/9810479. See also: I. Masina and C. A. Savoy, Nucl. Phys. B 661, 365 (2003) arXiv:hep-ph/0211283.

[17] See for example, R. Kitano, M. Koike and Y. Okada, Phys. Rev. D 66, 096002 (2002) arXiv:hep-ph/0203110; R. Kitano, M. Koike, S. Komine and Y. Okada, Phys. Lett. B 575, 300 (2003) arXiv:hep-ph/0308021 and references there in.

[18] K. S. Babu and C. Kolda, Phys. Rev. Lett. 89, 241802 (2002) arXiv:hep-ph/0206310; A. Dedes, J. R. Ellis and M. Raidal, Phys. Lett. B 549, 159 (2002) arXiv:hep-ph/0209207;

A. Brignole and A. Rossi, Phys. Lett. B 566, 217 (2003) arXiv:hep-ph/0304081.

[19] T. Blazek and S. F. King, Phys. Lett. B 518, 109 (2001) arXiv:hep-ph/0105005.

[20] M. L. Brooks et al. [MEGA Collaboration], Phys. Rev. Lett. 83 (1999) 1521 arXiv:hep-ex/9905013.

[21] K. Abe et al. [Belle Collaboration], arXiv:hep-ex/0310029 
[22] K. Inami, for the Belle Colloboration, Talk presented at the 19th International Workshop on Weak Interactions and Neutrinos (WIN-03) October 6th to 11th, 2003, Lake Geneva, Wisconsin, USA.

[23] Web page: http://meg.psi.ch

[24] [LEP Higgs Working Group for Higgs boson searches Collaboration], arXiv:hep-ex/0107029. LEPSUSYWG, ALEPH, DELPHI, L3 and OPAL experiments, note LEPSUSYWG/01-03 (http://lepSUSY.web.cern.ch/lepSUSY/Welcome.html).

[25] H. Baer, M. Drees, F. Paige, P. Quintana and X. Tata, Phys. Rev. D 61, 095007 (2000) arXiv:hep-ph/9906233 ; H. Baer, T. Krupovnickas and X. Tata, JHEP 0307, 020 (2003) arXiv:hep-ph/0305325.

[26] H. Baer, C. Balazs, A. Belyaev, T. Krupovnickas and X. Tata, JHEP 0306 (2003) 054 arXiv:hep-ph/0304303.

[27] F. Gabbiani, E. Gabrielli, A. Masiero and L. Silvestrini, Nucl. Phys. B 477, 321 (1996) arXiv:hep-ph/9604387.

[28] S. Bertolini, F. Borzumati, A. Masiero and G. Ridolfi, Nucl. Phys. B 353, 591 (1991); F. Borzumati, C. Greub, T. Hurth and D. Wyler, Phys. Rev. D 62, 075005 (2000) arXiv:hep-ph/9911245; M. Ciuchini, G. Degrassi, P. Gambino and G. F. Giudice, Nucl. Phys. B 534, 3 (1998) arXiv:hep-ph/9806308; G. Degrassi, P. Gambino and G. F. Giudice, JHEP 0012, 009 (2000) arXiv:hep-ph/0009337; M. Carena, D. Garcia, U. Nierste and C. E. M. Wagner, Phys. Lett. B 499, 141 (2001) arXiv:hep-ph/0010003.

[29] F. Borzumati, C. Greub and Y. Yamada, arXiv:hep-ph/0305063; F. Borzumati, C. Greub and Y. Yamada, arXiv:hep-ph/0311151.

[30] R. Barate et al. [ALEPH Collaboration], Phys. Lett. B 429 (1998) 169;

K. Abe et al. [Belle Collaboration], Phys. Lett. B 511 (2001) 151 arXiv:hep-ex/0103042; S. Chen et al. [CLEO Collaboration], Phys. Rev. Lett. 87 (2001) 251807 arXiv:hep-ex/0108032.

[31] Y. B. Dai, C. S. Huang and H. W. Huang, Phys. Lett. B 390, 257 (1997) [Erratum-ibid. B 513, 429 (2001)] arXiv:hep-ph/9607389]; C. S. Huang, W. Liao and Q. S. Yan, Phys. Rev. D 59, 011701 (1999) arXiv:hep-ph/9803460; S. R. Choudhury and N. Gaur, Phys. Lett. B 451, 86 (1999) arXiv:hep-ph/9810307; K. S. Babu and C. F. Kolda, Phys. Rev. Lett. 84, 228 (2000) arXiv:hep-ph/9909476; C. Bobeth, T. Ewerth, F. Kruger and J. Urban, Phys. Rev. D 64, 074014 (2001) arXiv:hep-ph/0104284.

[32] An incomplete list: C. S. Huang, W. Liao, Q. S. Yan and S. H. Zhu, Phys. Rev. D 63, 114021 (2001) [Erratum-ibid. D 64, 059902 (2001)] arXiv:hep-ph/0006250. A. Dedes, H. K. Dreiner and U. Nierste, Phys. Rev. Lett. 87, 251804 (2001) arXiv:hep-ph/0108037; R. Arnowitt, B. Dutta, T. Kamon and M. Tanaka, Phys. Lett. B 538, 121 (2002) arXiv:hep-ph/0203069; H. Baer, C. Balazs, A. Belyaev, J. K. Mizukoshi, X. Tata and Y. Wang, JHEP 0207, 050 (2002) arXiv:hep-ph/0205325; S. Baek, P. Ko and W. Y. Song, JHEP 0303, 054 (2003) arXiv:hep-ph/0208112 ; A. Dedes, J. R. Ellis and M. Raidal, Phys. Lett. B 549, 159 (2002) arXiv:hep-ph/0209207; R. Dermisek, S. Raby, L. Roszkowski and R. Ruiz De Austri, JHEP 0304, 037 (2003) arXiv:hep-ph/0304101; T. Blazek, 
S. F. King and J. K. Parry, arXiv:hep-ph/0308068; G. L. Kane, C. Kolda and J. E. Lennon, arXiv:hep-ph/0310042,

[33] J. L. Feng, K. T. Matchev and F. Wilczek, Phys. Lett. B 482, 388 (2000) arXiv:hep-ph/0004043.

[34] P. Gondolo, J. Edsjo, P. Ullio, L. Bergstrom, M. Schelke and E. A. Baltz, arXiv:astro-ph/0211238.

[35] J. Edsjo, M. Schelke, P. Ullio and P. Gondolo, JCAP 0304 (2003) 001 arXiv:hep-ph/0301106.

[36] G. Belanger, F. Boudjema, A. Pukhov and A. Semenov, Comput. Phys. Commun. 149 (2002) 103 arXiv:hep-ph/0112278.

[37] M. Kamionkowski and M. S. Turner, Phys. Rev. D 42 (1990) 3310.

[38] P. Salati, Phys. Lett. B 571 (2003) 121 arXiv:astro-ph/0207396.

[39] F. Rosati, Phys. Lett. B 570 (2003) 5 arXiv:hep-ph/0302159.

[40] S. Profumo and P. Ullio, JCAP 0311 (2003) 006 arXiv:hep-ph/0309220.

[41] J. R. Ellis, A. Ferstl and K. A. Olive, Phys. Lett. B 532 (2002) 318 arXiv:hep-ph/0111064. J. R. Ellis, A. Ferstl and K. A. Olive, Phys. Rev. D 63 (2001) 065016 arXiv:hep-ph/0007113;

[42] A. Bottino, F. Donato, N. Fornengo and S. Scopel, Phys. Rev. D 63 (2001) 125003 arXiv:hep-ph/0010203 ; L. Roszkowski, R. Ruiz de Austri and T. Nihei, JHEP 0108 (2001) 024 arXiv:hep-ph/0106334; U. Chattopadhyay, A. Corsetti and P. Nath, Phys. Rev. D 68 (2003) 035005 arXiv:hep-ph/0303201.

[43] V. Bertin, E. Nezri and J. Orloff, JHEP 0302 (2003) 046 arXiv:hep-ph/0210034.

[44] U. F. Katz [The ANTARES Collaboration], arXiv:astro-ph/0310736.

Antares web-page: http://www.antares.in2p3.fr

[45] F. Halzen and D. Hooper, JCAP 0401 (2004) 002 arXiv:astro-ph/0310152.

ICECUBE web-page: http://www.icecube.wisc.edu

[46] K. Griest and D. Seckel, Phys. Rev. D 43 (1991) 3191.

[47] C. Boehm, A. Djouadi and M. Drees, Phys. Rev. D $62 \quad$ (2000) 035012 arXiv:hep-ph/9911496.

[48] J. R. Ellis, K. A. Olive and Y. Santoso, Phys. Lett. B 539 (2002) 107 arXiv:hep-ph/0204192.

[49] J. R. Ellis, T. Falk, K. A. Olive and Y. Santoso, Nucl. Phys. B 652 (2003) 259 arXiv:hep-ph/0210205.

[50] S. Profumo, Phys. Rev. D 68 (2003) 015006 arXiv:hep-ph/0304071. 
[51] H. Baer, F. E. Paige, S. D. Protopescu and X. Tata, arXiv:hep-ph/0312045.

[52] J. Hisano, T. Moroi, K. Tobe and M. Yamaguchi, Phys. Lett. B 391, 341 (1997) [Erratumibid. B 397, 357 (1997)] arXiv:hep-ph/9605296.

[53] C. T. Hill, Phys. Lett. B 135 (1984) 47; J. R. Ellis, K. Enqvist, D. V. Nanopoulos and K. Tamvakis, Phys. Lett. B 155 (1985) 381; M. Drees, Phys. Lett. B 158 (1985) 409. For a recent analysis, see, for example: U. Chattopadhyay and D. P. Roy, Phys. Rev. D 68, 033010 (2003) arXiv:hep-ph/0304108.

[54] S. Profumo and C. E. Yaguna, arXiv:hep-ph/0307225, to appear on Nucl. Phys. B.

[55] H. Baer, A. Belyaev, T. Krupovnickas and X. Tata, arXiv:hep-ph/0311351. 